\title{
Endovaskuläre Eingriffe in der interventionellen Neuroradiologie
}

\author{
Neue Aspekte
}

\begin{abstract}
Die interventionelle Neuroradiologie (INR) zeichnet sich durch hohe Innovationsbereitschaft aus, und die Behandlungsmethoden entwickeln sich in immer kürzeren Abständen weiter. Der Anästhesist ist zu den verschiedensten Zeitpunkten mit Patienten, die an unterschiedlichsten neurovaskulären Erkrankungen leiden, konfrontiert. Daher ist es für ihn umso wichtiger, über neuroanästhesiologisches Wissen und aktuelle Kenntnisse der INR-Behandlungsmethoden zu verfügen.
\end{abstract}

\section{Hintergrund}

Endovaskuäre Eingriffe in der INR umfassen Eingriffe an den Hals-, Hirn- und Rückenmarkgefäßen. Prinzipiell unterscheidet man Interventionen, bei denen Gefäße eröffnet (Rekanalisation) oder verschlossen werden (Embolisation). Eingriffe an den Spinalgefäßen und extrakraniell können häufig ohne Anästhesie durchgeführt werden; intrakranielle Interventionen bedürfen nahezu immer der Anästhesie. Die Anästhesie hatte hierbei zunächst drei wesentliche Aufgaben [49]: Bereitstellung eines immobilen und physiologisch stabilen Patienten, Anpassung des Blutdrucks an die Erfordernisse des jeweiligen Eingriffs und Komplikationsmanagement. im Abschn. „Anästhesie" wird das anästhesiologische Aufgabengebiet dezidierter beschrieben und umfasst zusätzlich das Antikoagulations- management, die Überwachung der $\mathrm{Pa}$ tienten während des Transports und die rasche Beendigung der Narkose mit dem Ziel der neurologischen Beurteilung des Patienten unmittelbar nach dem Eingriff [20]. Seit 2007 wird die INR für die endovaskuläre Aneurysmabehandlung [30] als etabliertes Verfahren eingestuft. Die Anforderungen an die Anästhesie werden um Strahlenschutzaspekte erweitert [50].

Dem Zusammenwirken von Neuroanästhesie und INR wurde in der vorliegenden Zeitschrift Der Anaesthesist 2006 ein Leitthemenbeitrag gewidmet [35]. An der Vorbereitung, Narkoseführung und dem Monitoring sowie der Grundaussage, entscheidend zum technischen und klinischen Erfolg beizutragen [35], hat sich seither nichts geändert. Neu sind die Materialvielfalt und die exponentielle $\mathrm{Zu}$ nahme von Neurointerventionen, aber auch der Kliniken mit neurointerventionellem Schwerpunkt. Vor diesem Hintergrund gibt dieser Beitrag einen aktualisierten Überblick, speziell die neuen Aspekte der interventionellen Schlaganfallbehandlung, der endovaskulären Aneurysmabehandlung, des Strahlenschutzes und des Komplikationsmanagements betreffend.

\section{Grundlagen}

Von 1960 an wurden erste interventionelle Eingriffe vorgenommen, wie die transluminale Embolisation einer arteriovenösen Malformation (AVM) oder der Bal- lonverschluss zur Aneurysmabehandlung. Serbinenko [42] berichtete 1974 über 300 Patienten, die er seit 1963 mit Ballons behandelt hatte. Meilensteine der endovaskulären Therapie sind die erfolgreichen Behandlungen eines A.-basilarisKopfaneurysmas und eines supraklinoidalen A.-carotis-interna(ACI)-Aneurysmas mit ablösbaren Ballons durch Serbinenko.

\section{Aneurysmabehandlung mit Coils}

Eine neue Ära begann mit der Entwicklung elektrolytisch ablösbarer Platinspiralen („coils“, [19]), die 1990 erstmals bei einem Patienten mit Aneurysma eingesetzt wurden [18]. Seither haben sich Behandlungstechniken und Materialien rasant weiterentwickelt. Die letzten 15 Jahre der INR waren hinsichtlich der Aneurysmabehandlung geprägt durch die ballonassistierte „Remodeling“-Technik von Moret et al. [31], den Einsatz von intrakraniellen Stents zur Therapie bislang nichtbehandelbarer breitbasiger Aneurysmen und die Einführung von „flow diverter“, mit denen insbesondere Riesenaneurysmen ohne Coils behandelt werden.

Die Beschichtung von Coils sollte die Aneurysmaheilung befördern. Allerdings sind die ersten Studiendaten wenig überzeugend. Die Verwendung bioaktiver, resorbierbarer Substanzen zeigten bislang 
unveränderte oder sogar schlechtere klinische Ergebnisse als konventionelle Platinspiralen. Aktuell stehen Studienergebnisse zu neueren hydrogelbeschichteten Coils noch aus (German Randomized Endovascular Aneurysm Trial, GREAT). Aber nicht nur die Beschichtungen der Coils sind weiterentwickelt worden, sondern v. a. die Coils selbst. Es wurden neue u. a. 3D- Konfigurationen, unterschiedliche Härtegrade (steif, Standard, weich sowie ultraweich) und kleine Größen mit 1-mm-Durchmesser und 1-cm-Coil-Länge entwickelt; Ziel ist, die Aneurysmen noch dichter zu packen, um damit die Rezidivhäufigkeit $\mathrm{zu}$ reduzieren.

Vorangetrieben wurde diese Entwicklung durch die International Subarachnoid Aneurysm Trial (ISAT, [28]), in der Patienten mit Subarachnoidalblutung (SAB) und Nachweis von Aneurysmen, die mit beiden Methoden bei vergleichbarem Risiko hätten behandelt werden können, randomisiert wurden. Die Studie begann 1994, also kurze Zeit nach Einführung der ablösbaren Platinspiralen, und wurde 2002 vorzeitig abgebrochen, nachdem 2146 Patienten aufgenommen worden waren. Grund des Abbruchs war eine signifikant geringere Morbidität und Letalität ["modified Rankin Score" (mRS) $>2$ ] in der endovaskulär (23,7\%) gegenüber der mit Clip versorgten Gruppe $(30,6 \%,[28])$.

Der mRS ist eine in Schlaganfallstudien häufig verwendete Funktionsskala zur Erfassung des Beeinträchtigungsgrads in Bezug auf die Teilnahme am sozialen Leben. In einer Punktskala von 0 (keine Behinderung) bis 6 (Tod) wird beurteilt, inwieweit der Patient in der Lage ist, bestimmte Alltagsaktivitäten (z. B. Laufen und Körperpflege; • Tab. 1) auszuführen bzw. auf Hilfe angewiesen ist.

\section{Endovaskuläre Rekanalisation beim akuten Schlaganfall}

Die lokale Schlaganfallbehandlung hat sich durch zuletzt sehr gute Ergebnisse in nichtrandomisierten "Single-center"-Studien etabliert. Die lokale intraarterielle Lyse (LIF) wurde erstmals 1958 von Sussmann u. Fitch beschrieben (zit. nach [3]), die erfolgreich einen extrakraniellen ACI-Verschluss wiedereröffneten.

\title{
A. Berlis \\ Endovaskuläre Eingriffe in der interventionellen Neuroradiologie. Neue Aspekte
}

\begin{abstract}
Zusammenfassung
Die interventionelle Neuroradiologie (INR) hat sich in den letzten 10 Jahren insbesondere in der Aneurysmabehandlung und in der akuten ischämischen Schlaganfallbehandlung etabliert, sodass die Zahl der Eingriffe, aber auch die Standorte mit spezieller neuroradiologischer Expertise zugenommen haben. Viele Eingriffe sind mittlerweile als Routine einzuordnen, sodass auch Routine und Prozessoptimierung für die interdisziplinäre Zusammenarbeit zwischen Neuroradiologie und Anästhesie Voraussetzung für Patientenschutz und einen reibungslosen Ablauf sind. Routine bedeutet hierbei, dass das Wissen um die Erkrankung und die Art der Behandlung sowie die gegenseitigen Anforderun-
\end{abstract}

\section{Endovascular interventions in neuroradiology. New aspects}

\begin{abstract}
In the last 10 years interventional neuroradiology has become established especially in the endovascular treatment of aneurysms as the preferred treatment according to level 1 evidence and more recently in the treatment of acute ischemic stroke. These interventions are performed as routine procedures in an increasing number of centers. This, however, necessitates that anesthesiologists and neuroradiologists become increasingly more familiar with the complementary expectations and potential pitfalls in working together to
\end{abstract}

Seit den 1980er Jahren leistete die Gruppe um Zeumer Pionierarbeit in Bezug auf intrakranielle LIF und Lyseschemata bis hin zum „Bridging“-Therapieansatz [15]. Im Rahmen klinischer Heilversuche wurden von Zeumer et al. [51] die LIF sowohl im vorderen als auch im hinteren Stromkreislauf eingesetzt und erstbeschrieben. Aktuelle Ansätze in der Schlaganfallbehandlung zielen auf mechanische Systeme, die eine raschere Wiederherstellung der Durchblutung erlauben, da bisherige Analysen gezeigt haben, dass die Rekanalisation der beste Prädiktor eines guten klinischen Verlaufs ist.

Eine der letzten Entwicklungen, die eigentlich zur Stent-unterstützten CoilEmbolisation von Hirnaneurysmen geplant eingesetzt werden sollte, war zufäl- gen und Erwartungen von Interventionalist und Anästhesist bekannt sind. Zu differenzieren gilt der Notfalleingriff, bei dem die zeitlichen Abläufe kurz gehalten werden müssen, von dem elektiven Eingriff, der geplant unter optimierten Bedingungen ablaufen kann. Im vorliegenden Beitrag werden allgemeine Voraussetzungen hierfür benannt. Diskutiert werden die zwei häufigsten Interventionen: Behandlungen von Aneurysmen und Rekanalisationsbehandlungen beim akuten Schlaganfall.

\begin{abstract}
maximise efficiency and patient safety. As a further challenge there are different aspects to be considered in elective procedures and emergency procedures where time is brain. This article highlights these aspects for the two most common procedures: aneurysm treatment and multimodal recanalization treatment for acute ischemic stroke.
\end{abstract}

lig beim Bergen von Butgerinnseln erfolgreich. Bei diesem „stent retrieving“ wird ein selbstexpandierender Stent im verschlossenen Gefäß platziert und nicht abgelöst. Initial führt dies in der Regel zur Reperfusion des nachgeschalteten Hirngewebes, sodass die Ischämietoleranz des Gehirns verlängert wird. Nach 3-5 min dringt das Blutgerinnsel durch die StentMaschen in den Stent ein und bewirkt häufig einen Reverschluss, sodass der Stent mit dem Blutgerinnsel nach 5- bis 10-minütiger Verweildauer herausgezogen werden kann. Mit dieser Methode sind $80-90 \%$ der Verschlüsse rekanalisierbar.

Stents zur elektiven Behandlung symptomatischer intrakranieller Gefäßstenosen bieten eine Möglichkeit, sowohl in 


\begin{tabular}{|lll}
\hline Tab. 1 & Modifizierter Rankin-Score (mRS) \\
\hline Grad & $\begin{array}{l}\text { Ausmaß der Behinde- } \\
\text { rung }\end{array}$ & Bedeutung \\
\hline 0 & Keine Symptome & \\
\hline 1 & Geringe Symptome & $\begin{array}{l}\text { Alle üblichen Aufgaben und Aktivitäten können erfüllt } \\
\text { werden }\end{array}$ \\
\hline 2 & Leichte Behinderung & Patient ist in der Lage, sich ohne Hilfe zu versorgen \\
\hline 3 & Mäßige Behinderung & Patient benötigt Hilfe im Alltag. Gehen ist möglich \\
\hline 4 & Schwere Behinderung & $\begin{array}{l}\text { Patient benötigt Hilfe beim Gehen und kann sich nicht } \\
\text { selbst versorgen }\end{array}$ \\
\hline 5 & Sehr schwere Behinderung & $\begin{array}{l}\text { Bettlägriger Patient, inkontinent und vollständig pfle- } \\
\text { gebedürftig }\end{array}$ \\
\hline 6 & Tod & \\
\hline
\end{tabular}

der Akutphase als auch bei medikamentös refraktären Stenosen eine endovaskuläre Behandlung durchzuführen. Die unerwartet ungünstigen Ergebnisse der Studie Stenting and Aggressive Medical Management for Preventing Recurrent stroke in Intracranial Stenosis (SAMMPRIS, [8]) waren u. a. auf schlechte und unkritische Indikationen, auf geringe Einschlusszahlen pro behandelndes Zentrum und auf geringe Anforderungsprofile für die Qualifikation der Interventionalisten zurückzuführen. In dieser Studie konnte allerdings gezeigt werden, dass eine aggressive medikamentöse Therapie bessere Ergebnisse erzielt, als dies aus den bisherigen Studien Warfarin-Aspirin Symptomatic Intracranial Disease (WASID, [9]) bekannt war, sodass die Indikation zur Behandlung einer intrakraniellen Stenose noch kritischer als zuvor gesehen werden muss. Nach wie vor besteht eine Indikation, wenn die klinischen Symptome therapierefraktär oder die Stenose hämodynamisch relevant ist. Da es sich um seltene Eingriffe handelt, sollten diese an „High-volume“-Zentren durchgeführt werden, damit das prozedurale Risiko nicht durch eine Lernkurve unnötig erhöht wird.

\section{Arteriovenöse Gefäßfehlbildungen}

Die Behandlung arteriovenöser Gefäßfehlbildungen ist durch die Einführung neuer zugelassener Flüssigembolisate wie z. B. Onyx (Fa. ev3-Covidien) mit einer höheren Rate kompletter Verschlüsse verbunden. Zusätzliche technische Weiterentwicklungen des Kathetermaterials mit ablösbaren Katheterspitzen zwischen
1,5 und $5 \mathrm{~cm}$, die im Körper verbleiben können, bringen mehr Sicherheit bei der Behandlung.

\section{Technische Innovationen}

Beträchtlichen Einfluss auf die Interventionsqualität hat die Weiterentwicklung der Angiographieanlagen mit 3Dund Computertomographie(CT)-ähnlichen Funktionen. Niedrigdosisprogramme ohne wesentliche Qualitätsreduktion sind für den Strahlenschutz des Patienten und des Personals wesentlich.

Computertomographieähnliche Bilder können z. B. als Dyna- (Fa. Siemens, Erlangen) oder Xper-CT (Fa. Philips, Niederlande) intraprozedural akquiriert werden und erlauben, Komplikationen wie Hirnblutungen direkt in der Angiographieeinheit zu erkennen und therapeutische Schritte umgehend einzuleiten. Aktuell gibt es Bestrebungen, die Schlaganfalldiagnostik direkt in die Angiographieanlage zu verlagern und eine DynaCT, gefolgt von Dyna-CT-Angiographie und Dyna-CT-Perfusionsbildgebung mithilfe i.v.-Kontrastmittel-Gabe durchzuführen, um dann direkt mit der Intervention zu beginnen.

\section{Anästhesie}

Der Anästhesist ist zu den verschiedensten Zeitpunkten mit Patienten, die an unterschiedlichsten neurovaskulären Erkrankungen leiden, konfrontiert. Der neurointerventionelle Eingriff betrifft nur einen Ausschnitt des Managements. Aus Sicht des Interventionalisten ist für diese Momentaufnahme wichtig, dass der Patient während des Eingriffs in tiefer Narkose und relaxiert ist, während er im Anschluss an die Behandlung so schnell wie möglich extubiert werden sollte, damit sofort ein neurologischer Status erhoben werden kann. Dieses Management ist bei elektiven Patienten eher zu erreichen als im Rahmen von Notfallbehandlungen, bei denen die Narkoseeinleitung auch in der Akutphase und unter Zeitdruck rasch sowie sicher erfolgen muss.

\section{Notfallversorgung}

Entscheidend in der Akutphase ist zunächst, ob ein Eingriff in Allgemeinanästhesie mit Intubation (ITN) oder unter "stand-by" durchgeführt werden soll. Das Gebot der Zeitoptimierung gilt sowohl für die Diagnostik als auch die Behandlung. In - Tab. 2 sind die Anästhesiezeiten anhand von Schlaganfallpatienten am Freiburger Universitätsklinikum zwischen 1995 und 1999 sowie 2000 und 2002 aufgeführt [3]. Das Jahr 2000 wurde als Trennlinie benutzt, da zu diesem Zeitpunkt die „stroke unit" initiiert wurde. Der Zeitraum Anästhesie ist „bezogen auf Behandlung in Intubationsnarkose" mit 90 bzw. 85 min konstant, während Anästhesie-Stand-by im Median $48 \mathrm{~min}$ (1995-1999) bzw. 87 min (2000-2002) benötigte. Für den Zeitraum 2000-2002 ergibt sich folglich kein zeitlicher Vorteil zwischen Stand-by und ITN.

Im Zeitraum 2004-2005 konnte bei 26 Patienten, die eine „Bridging“-Behandlung, also eine kombinierte i.v.- und i.a.-Lyse erhielten, die Anästhesiezeit auf im Median 52 min reduziert werden. Diese Daten werden durch eine Publikation unterstützt, in der die Anästhesiezeit, und zwar der Zeit zwischen CT oder Magnetresonanztomogramm (MRT) und erster Gefäßdarstellung 50 min ohne und 65 min mit ITN betrug [6].

Der zeitliche Aufwand ist somit für eine ITN nicht wesentlich höher ist als für Stand-by. Für die Durchführung einer Narkose spricht, dass sich der Blutdruck besser steuern lässt und der $\mathrm{Pa}$ tient ruhig liegt, sodass ein sicheres $\mathrm{Na}$ vigieren der Mikrokatheter durch die Hirngefäße möglich wird. Dies reduziert Komplikationen wie Gefäßdissektionen und Perforationen mit ggf. fataler intra- 
Tab. 2 Anästhesiezeiten bei Patienten mit Schlaganfall: zeitliche Abläufe zwischen Schlaganfallbeginn und Behandlung

\begin{tabular}{|c|c|c|c|c|c|c|c|}
\hline & & \multirow{2}{*}{$\begin{array}{l}\text { Anzahl der } \\
\text { Patienten } \\
\text { (Zeiten be- } \\
\text { kannt) }\end{array}$} & \multirow{2}{*}{$\begin{array}{l}\text { Mittelwert } \\
\text { (h, Minimum-Maxi- } \\
\text { mum) }\end{array}$} & \multirow{2}{*}{$\begin{array}{l}\text { Stan- } \\
\text { dardab- } \\
\text { weichung } \\
\text { (h) }\end{array}$} & \multirow{2}{*}{$\begin{array}{l}\text { Median } \\
\text { (h) }\end{array}$} & \multicolumn{2}{|l|}{ Patienten } \\
\hline & & & & & & $\begin{array}{l}\text { 1995-1999 } \\
\text { (Median) }\end{array}$ & $\begin{array}{l}\text { 2000-2002 } \\
\text { (Median) }\end{array}$ \\
\hline \multirow{2}{*}{$\begin{array}{l}\text { Ende der Bild- } \\
\text { gebung - Be- } \\
\text { ginn der Angio- } \\
\text { graphie }\end{array}$} & Anästhesie & 22 & 01:43 (00:25-03:32) & $00: 58$ & $01: 26$ & $01: 30$ & $01: 25$ \\
\hline & $\begin{array}{l}\text { "Anästhesie- } \\
\text { Stand-by" }\end{array}$ & $7(6)$ & 01:06 (00:42-01:55) & 00:29 & 00:57 & $00: 48$ & 01:27 \\
\hline
\end{tabular}

kranieller Blutung. Bei mechanischer Rekanalisation mit Stent retrieving kommt hinzu, dass beim Entfernen des Thrombus Zug auf die Hirngefäße ausgeübt wird, der über die leptomeningeale Reizung als sehr schmerzhaft empfunden wird und über trigeminovagale Reflexe sogar einen Herzstillstand auslösen kann.

\section{Intubationsnarkose vs.}

Lokalanästhesie in Sedierung

In verschiedenen Studien konnte gezeigt werden, dass die ITN gegenüber Stand-by-Eingriffen in Lokalanästhesie und Sedierung mit schlechteren kli- nischen Verläufen einhergehen $[1,12,16$, 21, 22, 23, 32]. Hassan et al. [21] berichten über 136 endovaskuläre Schlaganfallbehandlungen, bei denen in $23 \%$ der intubierten und in $14 \%$ der nichtintubierten Patienten Aspirationspneumonien auftraten. Ein schlechter klinischer Verlauf fand sich bei $83 \%$ der intubierten und bei 55\% der nichtintubierten Patienten. Vergleichbare Ergebnisse ergab die Arbeit von Jumaa et al. [23] mit signifikant höherer Rate an Pneumonien bei intubierten Patienten: 30 vs. 13,7\%. Nach Hassan et al. [21] ist die höhere Rate von schlechten klinischen Verläufen nicht auf die ITN und Pneumonie zurückzuführen.
Auffallend ist eine Zunahme der ITN bei zunehmender Schwere des Schlaganfalls.

Davis at al. [12] berichten über einen signifikant besseren klinischen Verlauf bei nichtintubierten Patienten von 60 vs. 15\%. Einschränkend ist zu dieser Analyse anzumerken, dass bei $40 \%$ der intubierten Patienten das erkrankte Gefäßterritorium die A. basilaris und bei $60 \%$ die mittlere Hirnarterie betraf, während bei den nichtintubierten Patienten 21\% der Gefäßverschlüsse in der A. basilaris und $79 \%$ in der mittleren Hirnarterie lokalisiert waren. Der natürliche Verlauf und die Behandlungsergebnisse sind bei Gefäßverschlüssen in diesen beiden Gefäß- 
Tab. 3 Endovaskuläre Eingriffe und mögliche Komplikationen

\begin{tabular}{|c|c|c|c|c|}
\hline Prozedur & Dringlichkeit der Behandlung & Komplikation & Diagnostik & Therapeutische Konsequenz \\
\hline $\begin{array}{l}\text { Aneurys- } \\
\text { ma }\end{array}$ & $\begin{array}{l}\text { Bei SAB so früh wie möglich, da } \\
\text { hohe Nachblutungsrate inner- } \\
\text { halb der ersten 6-12 h. Konsens } \\
\text { besteht darüber, dass nachts } \\
\text { schlechtere Behandlungsbedin- } \\
\text { gungen bestehen und deshalb } \\
\text { besser in der Routinearbeitszeit } \\
\text { behandelt werden sollte. Aus- } \\
\text { nahme und Indikation für akute } \\
\text { Behandlung: neurologisches } \\
\text { Defizit durch Vasospasmen oder } \\
\text { rezidivierende Blutungsereignisse }\end{array}$ & $\begin{array}{l}\text { Reruptur, Hyperkoagulabilität mit } \\
\text { Gefahr der Thrombusbildung und } \\
\text { Ischämie bei SAB, seltener bei } \\
\text { elektiven Eingriffen }\end{array}$ & $\begin{array}{l}\text { Intraprozedurales Flachdetek- } \\
\text { tor-CT (z. B. Dyna-CT, Fa. Sie- } \\
\text { mens, Erlangen, oder Xper-CT, } \\
\text { Fa. Philips, Niederlande) }\end{array}$ & $\begin{array}{l}\text { Operation mit Evaluation einer } \\
\text { raumfordernden Blutung, } \\
\text { Antikoagulation und Anti- } \\
\text { aggregation bei Thrombus mit } \\
\text { Eskalation von Heparin zu Aspi- } \\
\text { rin bis hin zu Glykoprotein-Ila/ } \\
\text { Illb-Inhibitoren }\end{array}$ \\
\hline $\begin{array}{l}\text { Arterio- } \\
\text { venöse } \\
\text { Malfor- } \\
\text { mation }\end{array}$ & $\begin{array}{l}\text { Elektiv: zeitnahe Behandlung } \\
\text { bei rezidivierenden Blutungs- } \\
\text { ereignissen oder bei aneurysma- } \\
\text { assoziierten Blutungen. Akut bei } \\
\text { raumfordernden Blutungen, ggf. } \\
\text { mit präoperativer Embolisation }\end{array}$ & $\begin{array}{l}\text { Intra- und v. a. postprozedurale } \\
\text { Blutung durch Umstellung der } \\
\text { Hämodynamik oder venösen } \\
\text { Stauungsblutungen, z. B. durch } \\
\text { Verschluss einer Drainagevene } \\
\text { oder Embolisatverschleppung in } \\
\text { die venöse Leiter (Risiko inner- } \\
\text { halb von } 24 \mathrm{~h} \text { nach Eingriff am } \\
\text { höchsten) }\end{array}$ & $\begin{array}{l}\text { Intraprozedurales Flachdetek- } \\
\text { tor-CT bei Blutungsverdacht. } \\
\text { Ansonsten Flachdetektor oder } \\
\text { CT-Kontrolle nach dem Eingriff } \\
\text { zur Detektion von Embolisat im } \\
\text { venösen System und Frage nach } \\
\text { intrazerebraler Blutung }\end{array}$ & $\begin{array}{l}\text { Operation bei Blutung. Antiko- } \\
\text { agulation zur Prävention einer } \\
\text { zerebralen Venenthrombose } \\
\text { und einer Stauungsblutung. } \\
\text { Dauer der Antikoagulation ab- } \\
\text { hängig vom CT-Befund }\end{array}$ \\
\hline $\begin{array}{l}\text { Arterio- } \\
\text { venöse } \\
\text { Fistel }\end{array}$ & Elektiv & $\begin{array}{l}\text { Verschleppung von Embolisat in } \\
\text { das Sinus- und Venensystem mit } \\
\text { Gefahr einer venösen Stauungs- } \\
\text { blutung im Gehirn }\end{array}$ & $\begin{array}{l}\text { CT-Kontrolle nach Eingriff zum } \\
\text { Ausschluss einer Blutung und } \\
\text { eines venösen Infarkts }\end{array}$ & Operation bei Blutung \\
\hline Epistaxis & $\begin{array}{l}\text { Akut bei hämoglobinwirksamem } \\
\text { Blutverlust }\end{array}$ & $\begin{array}{l}\text { Partikelverschleppung in das } \\
\text { Hirngefäßsystem über Anastomo- } \\
\text { sen von extra- nach intrakraniell }\end{array}$ & $\begin{array}{l}\text { Überwachung des meist wa- } \\
\text { chen Patienten }\end{array}$ & \\
\hline $\begin{array}{l}\text { Gefäßver- } \\
\text { schluss }\end{array}$ & Elektiv & $\begin{array}{l}\text { Schlaganfall durch Verschlep- } \\
\text { pung von Butgerinnsel oder } \\
\text { hämodynamisch durch Minder- } \\
\text { perfusion des ipsilateralen nach- } \\
\text { geschalteten Stromgebiets }\end{array}$ & $\begin{array}{l}\text { Temporärer Okklusionstest vor } \\
\text { permanentem Veschluss, Blut- } \\
\text { druckmessung }\end{array}$ & $\begin{array}{l}\text { Ggf. Blutruckanhebung nach } \\
\text { Gefäßverschluss }\end{array}$ \\
\hline $\begin{array}{l}\text { Karotis- } \\
\text { Stent }\end{array}$ & $\begin{array}{l}\text { Elektiv bei Stenosen, akut bei } \\
\text { frischem schwerem Schlaganfall } \\
\text { z. B. mit Karotisverschluss }\end{array}$ & $\begin{array}{l}\text { Bradykardie oder Herzstillstand } \\
\text { während PTA des Karotisbulbus } \\
\text { Zeitversetzt Hyperperfusionssyn- } \\
\text { drom + Reperfusionsblutung }\end{array}$ & $\begin{array}{l}\text { EKG, postprozedurales Flachde- } \\
\text { tektor-CT oder konventionelles } \\
\text { CT }\end{array}$ & $\begin{array}{l}\text { Atropin vor der PTA und bei Be- } \\
\text { darf Dosiserhöhung. Blutdruck- } \\
\text { senkung nach Stenting auf } \\
\text { systolische Werte } \leq 140 \mathrm{mmHg}\end{array}$ \\
\hline "Stroke" & Perakut:„Time is brain“ & $\begin{array}{l}\text { Gefäßverletzung, Dissektion, } \\
\text { Ruptur mit SAB oder Hirnparen- } \\
\text { chymblutung }\end{array}$ & $\begin{array}{l}\text { Postprozedurales Flachdetek- } \\
\text { tor-CT oder konventionelles CT }\end{array}$ & $\begin{array}{l}\text { Dekompression bei großem } \\
\text { raumfordernd wirkendem } \\
\text { Schlaganfall }\end{array}$ \\
\hline $\begin{array}{l}\text { Tumo- } \\
\text { remboli- } \\
\text { sation }\end{array}$ & Elektiv präoperativ & $\begin{array}{l}\text { Partikelverschleppung in das } \\
\text { Hirngefäßsystem über Anastomo- } \\
\text { sen von extra- nach intrakraniell }\end{array}$ & $\begin{array}{l}\text { Überwachung des meist wa- } \\
\text { chen Patienten }\end{array}$ & \\
\hline $\begin{array}{l}\text { Vaso- } \\
\text { spasmen }\end{array}$ & $\begin{array}{l}\text { Akut, wenn klinische Symptome } \\
\text { mit neurologischen Ausfällen } \\
\text { bestehen }\end{array}$ & $\begin{array}{l}\text { Verwendung von Vasodilatanz- } \\
\text { ien geht mit mitunter massiven } \\
\text { Blutdruckabfällen einher, denen } \\
\text { entgegengewirkt werden muss. } \\
\text { Bei PTA der Gefäße Gefahr der } \\
\text { Gefäßruptur mit Hirnblutung }\end{array}$ & $\begin{array}{l}\text { Blutdruckmessung invasiv, } \\
\text { Flachdetektor-CT oder konven- } \\
\text { tionelles CT }\end{array}$ & $\begin{array}{l}\text { Katecholamine bei Be- } \\
\text { darf. Hypertonie mit MAP } \\
\text { >95 mmHg gewünscht. Ggf. } \\
\text { Operation mit Hämatomaus- } \\
\text { räumung }\end{array}$ \\
\hline $\begin{array}{l}\text { Spinale } \\
\text { Angio- } \\
\text { graphie }\end{array}$ & Elektiv & $\begin{array}{l}\text { Verschluss der A. spinalis anterior } \\
\text { oder posterior mit Querschnitts- } \\
\text { lähmung }\end{array}$ & $\begin{array}{l}\text { Überwachung des meist wa- } \\
\text { chen Patienten }\end{array}$ & $\begin{array}{l}\text { Reduktion der Darmblähung } \\
\text { durch abführende Maßnahmen } \\
\text { am Tag zuvor und Buscupan i.v. } \\
\text { während der Angiographie }\end{array}$ \\
\hline $\begin{array}{l}\text { Spinale } \\
\text { Interven- } \\
\text { tion }\end{array}$ & $\begin{array}{l}\text { Elektiv, Ausnahme: akut dekom- } \\
\text { pensierte arteriovenöse Gefäß- } \\
\text { fehlbildung }\end{array}$ & $\begin{array}{l}\text { Verschluss der A. spinalis anterior } \\
\text { oder posterior mit Querschnitts- } \\
\text { lähmung }\end{array}$ & $\begin{array}{l}\text { Überwachung des wachen oder } \\
\text { intubierten Patienten }\end{array}$ & $\begin{array}{l}\text { Reduktion der Darmblähung } \\
\text { durch abführende Maßnahmen } \\
\text { am Tag zuvor und Buscupan i.v. } \\
\text { während der Angiographie }\end{array}$ \\
\hline
\end{tabular}


Tab. 4 Augsburger Antiaggregations- und Antikoagulationsschema

\begin{tabular}{|c|c|c|c|c|}
\hline & \multicolumn{2}{|l|}{ Heparin } & \multirow{2}{*}{$\begin{array}{l}\text { Aspirin und Clopidogrel/Ticagre- } \\
\text { lor/Prasugrel }\end{array}$} & \multirow[t]{2}{*}{ Langzeitmedikation } \\
\hline & Intraprozedural & Postprozedural & & \\
\hline $\begin{array}{l}\text { Aneurysma bei } \\
\text { SAB }\end{array}$ & $\begin{array}{l}\text { Nach 1. Coil mit } 5000 \text { IE i.v. } \\
\text { als Bolus }\end{array}$ & $\begin{array}{l}48 \mathrm{~h} \text { mit PTT-Zielwert } \\
50-60 \mathrm{~s}\end{array}$ & - & - \\
\hline $\begin{array}{l}\text { Aneurysma, in- } \\
\text { zidentell }\end{array}$ & $\begin{array}{l}5000 \text { IE i.v. als Bolus nach } \\
\text { Anlage der Schleuse }\end{array}$ & $\begin{array}{l}48 \mathrm{~h} \text { mit PTT-Zielwert } \\
50-60 \mathrm{~s}\end{array}$ & - & - \\
\hline $\begin{array}{l}\text { Aneurysma mit } \\
\text { Stent und Coils }\end{array}$ & $\begin{array}{l}5000 \text { IE i.v. als Bolus nach } \\
\text { Anlage der Schleuse }\end{array}$ & $\begin{array}{l}48 \mathrm{~h} \text { mit PTT-Zielwert } \\
40-50 \mathrm{~s}\end{array}$ & $\begin{array}{l}\text { Prämedikation mit ASS } 100 \mathrm{mg} \\
\text { 1-0-0 und Clopidogrel } 75 \mathrm{mg} \text { 0-0-1 } \\
5 \text { Tage vor dem Eingriff } \\
\text { Bei Nonresponder im Multiplate- } \\
\text { Test Wechsel auf Brilique }^{\oplus} \text { oder } \\
\text { Prasugrel }^{\circledR}\end{array}$ & $\begin{array}{l}\text { ASS } 100 \text { mg für } 6 \text { Monate, dann nach } \\
\text { Angiographiebefund komplett ab- } \\
\text { setzen } \\
\text { Clopidogrel } 75 \text { mg oder alternative } \\
\text { Präparate für } 6 \text { Wochen }\end{array}$ \\
\hline $\begin{array}{l}\text { Aneurysma mit } \\
\text { „flow diverter" }\end{array}$ & $\begin{array}{l}5000 \text { IE i.v. als Bolus nach } \\
\text { Anlage der Schleuse }\end{array}$ & $\begin{array}{l}48 \mathrm{~h} \text { mit PTT-Zielwert } \\
40-50 \mathrm{~s}\end{array}$ & $\begin{array}{l}\text { Prämedikation mit ASS } 100 \mathrm{mg} \\
\text { 1-0-0 und Clopidogrel } 75 \mathrm{mg} \text { 0-0-1 } \\
5 \text { Tage vor dem Eingriff } \\
\text { Bei Nonresponder im Multiplate- } \\
\text { Test Wechsel auf Brilique }^{\oplus} \text { oder } \\
\text { Prasugrel }^{\circledR}\end{array}$ & $\begin{array}{l}\text { ASS } 100 \text { mg für } 1 \text { Jahr und dann nach } \\
\text { Angiographiebefund komplett ab- } \\
\text { setzen } \\
\text { Clopidogrel } 75 \text { mg oder alternative } \\
\text { Präparate für } 3 \text { Monate }\end{array}$ \\
\hline $\begin{array}{l}\text { Stent extra-/int- } \\
\text { rakraniell, Notfall }\end{array}$ & $\begin{array}{l}5000 \text { IE i.v. als Bolus nach } \\
\text { Anlage der Schleuse }\end{array}$ & - & $\begin{array}{l}\text { „Loading dose“ mit } 600 \text { mg Clopi- } \\
\text { dogrel p.o. oder über Magensonde } \\
\text { und ASS } 500 \text { mg i.v. } \\
\text { Bei Nonresponder im Multiplate- } \\
{\text { Test Wechsel auf Brilique }{ }^{\circledast} \text { oder }} \\
\text { Prasugrel }^{\circledast}\end{array}$ & $\begin{array}{l}\text { ASS } 100 \mathrm{mg} \text { zeitlebens und Clopidog- } \\
\text { rel } 75 \mathrm{mg} \text { oder alternative Präparate } \\
\text { für } 3 \text { Monate }\end{array}$ \\
\hline $\begin{array}{l}\text { Stent extra-/ } \\
\text { intrakraniell, } \\
\text { elektiv }\end{array}$ & $\begin{array}{l}5000 \text { IE i.v. als Bolus nach } \\
\text { Anlage der Schleuse }\end{array}$ & - & $\begin{array}{l}\text { Prämedikation mit ASS } 100 \mathrm{mg} \\
\text { 1-0-0 und Clopidogrel } 75 \mathrm{mg} \text { 0-0-1 } \\
5 \text { Tage vor dem Eingriff } \\
\text { Bei Nonresponder im Multiplate- } \\
\text { Test Wechsel auf Brilique }^{\circledast} \text { oder } \\
\text { Prasugrel }^{\circledR}\end{array}$ & $\begin{array}{l}\text { ASS } 100 \mathrm{mg} \text { zeitlebens und Clopidog- } \\
\text { rel } 75 \mathrm{mg} \text { oder alternative Präparate } \\
\text { für } 6 \text { Wochen }\end{array}$ \\
\hline AVM oder AVF & & $\begin{array}{l}\text { Heparin mit PTT-Ziel- } \\
\text { wert } 40-50 \text { s für } 48 \mathrm{~h} \\
\text { zur Vermeidung venöser } \\
\text { Thrombosen nach } \\
\text { Embolisation, ggf. LMW- } \\
\text { Heparin für Wochen/ } \\
\text { Monate }\end{array}$ & - & - \\
\hline
\end{tabular}

ASS Acetylsalicylsäure, AVF arteriovenöse Fistel, AVM arteriovenöse Malformation, LMW "low molecular weight", PTT "partial thromboplastin time" (partielle Thromboplastinzeit), $S A B$ Subarachnoidalblutung.

territorien zu unterschiedlich, als dass man diese gleichsetzen könnte.

Vor- und Nachteile der ITN und der Lokalanästhesie mit Sedierung wurden von Flexman et al. [16] zusammengefasst. Vorteile der ITN liegen u. a. im bewegungslosen Patienten, höherem $\mathrm{Pa}$ tientenkomfort sowie der Kontrolle von Oxygenierung und Beatmung. Nachteile sind u. a. das höhere Risiko einer Pneumonie und das Risiko einer Hypotension, die von Davis et al. [12] bei einem systolischen Blutdruck $<140 \mathrm{mmHg}$ als Prädiktor für den schlechten klinischen Verlauf ausgemacht wurden. Vorteile der Lokalanästhesie mit Sedierung sind u. a. die kontinuierliche Beurteilbarkeit des neurologischen Status und seltenere hypotensive Blutdruckwerte, die ggf. mit einer Abnahme der Hirndurchblutung einhergehen. Nachteile sind die Risiken einer Hyperkapnie und Hypoxie, die Aspiration durch ungesicherte Atemwege, geringer Patientenkomfort und fehlendes Verständnis des Patienten mit zunehmendem Schweregrad des Schlaganfalls.

Voraussetzungen für ein gutes Ergebnis sind neben dem engen Zeitfenster das optimale Blutdruckmanagement mit Blutdruckwerten $\geq 140 \mathrm{mmHg}$ zur Aufrechterhaltung der leptomeningealen Kollateralendurchblutung vor und während der Behandlung sowie eine Bludrucksenkung $<140 \mathrm{mmHg}$ nach Wiedereröffnung des Gefäßes, um Reperfusionsschäden zu vermeiden.
Derzeit kann aufgrund der Datenlage keine abschließende Empfehlung für oder gegen eine ITN bei der akuten Schlaganfallbehandlung gegeben werden. Fehlende eindeutige Angaben zu den durchgeführten Sedierungen unterstreichen die Forderung nach einer prospektiven randomisierten Studie. Aus eigener Sicht scheinen die Vorteile einer ITN zu überwiegen.

\section{Narkoseeinleitung und Patientenvorbereitung}

Konsequenterweise ist die Optimierung des Zeitablaufs der Narkoseeinleitung oder der Lokalanästhesie in Sedierung anzustreben. Diese beginnt beim Schlaganfallpatienten durch die schnellstmög- 

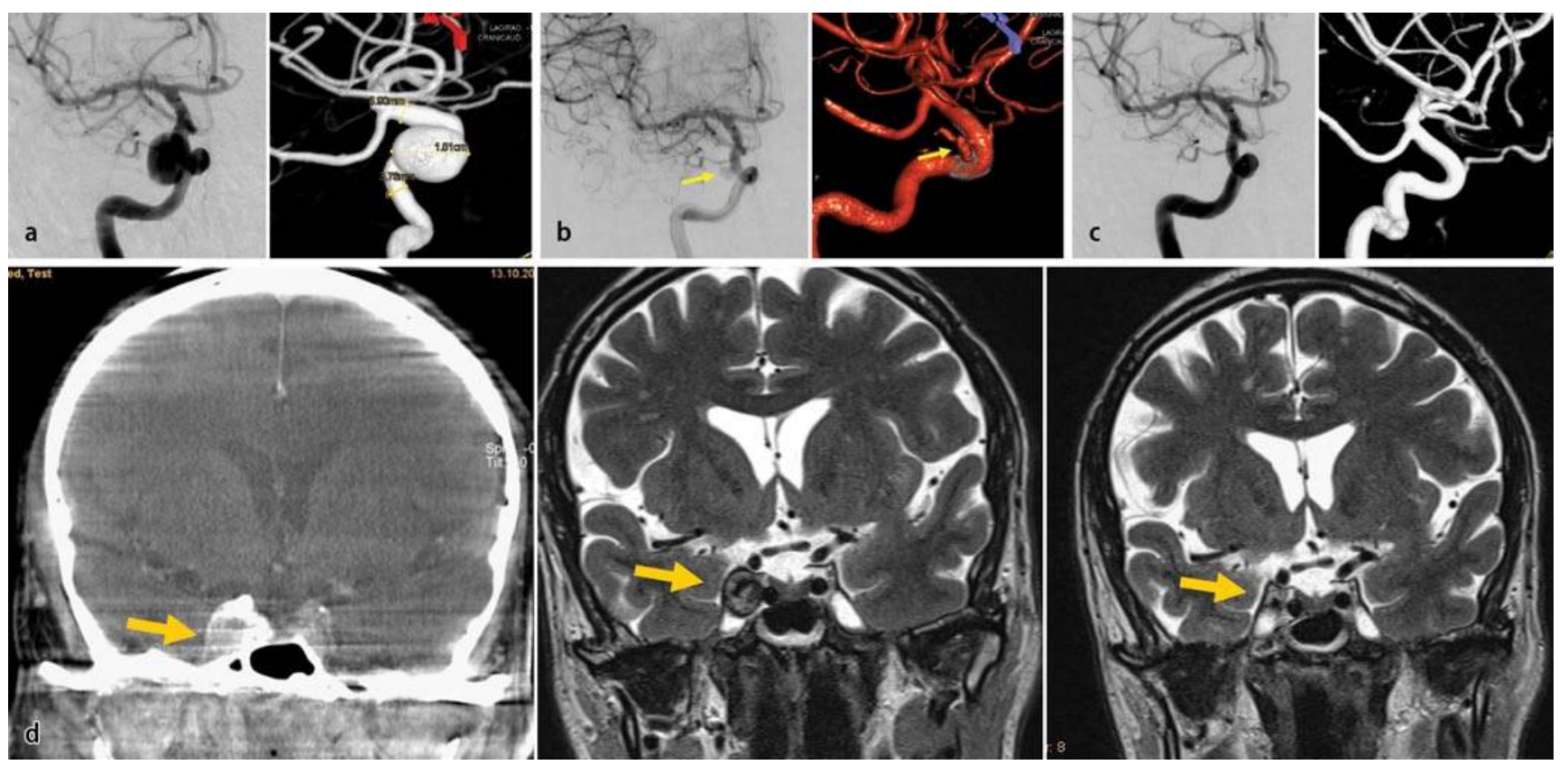

Abb. $1 \Delta$ Ein 62-jähriger Patient mit rechtsseitigem symptomatischem kavernösem A.-carotis-interna(ACI)-Aneurysma. Es besteht seit mehreren Wochen eine komplette Ophthalmoplegie. a, links p.-a.-Strahlengang der konventionellen digitalen Subtraktionsangiographie, rechts seitliche 3D-Darstellung. b Kontrolle 6 Wochen nach Behandlung des Aneurysmas ausschließlich mit einem „flow diverter" und ohne Platinspiralen. Nachweis eines kleinen Rests (gelber Pfeil) bei deutlich rückläufigen klinischen Symptomen. c Kontrolle 6 Monate nach Behandlung. Kompletter Verschluss des Aneurysmas. d links Postprozedurales Dyna-Computertomogramm mit koronarer Rekonstruktion und 5-mm-Schichtdicke. In der Mitte und rechts jeweils koronare $\mathrm{T}_{2}$-gewichtete Darstellung und 2,5-mm-Schichtdicke nach 6 Wochen und 6 Monaten. Das kavernöse ACl-Aneurysma (gelber Pfeil) ist im Verlauf komplett verschlossen und hat sich vollständig zurückgebildet

liche Verfügbarkeit eines Anästhesieteams, gefolgt von einem zügigen Transport vom CT oder MRT in die Angiographie-Suite mit lokal verfügbarem betriebsbereitem Anästhesiegerät. Die Narkoseeinleitung kann beschleunigt werden, wenn der arterielle Zugang in der Leiste gemeinsam genutzt wird. Eine um $1 \mathrm{~F}$ größere Schleuse, als sie für den Eingriff benötigt wird, ist zur Blutdruckmessung ausreichend, die meist über eine 7-F-Schleuse und über den Seitarm der Schleuse erfolgen kann.

Bei Patienten mit Bridging-Behandlung, also Patienten, die nach dem Blutungsausschluss im CT bereits mit „recombinant tissue-type plasminogen activator" (rt-PA) oder Glykoprotein(GP)IIa/IIIb-Hemmer vorbehandelt sind, besteht ein höheres Blutungsrisiko im Rahmen der arteriellen und venösen Gefäßpunktion. Hinzu kommt der Zeitdruck mit dem Risiko eines um $10 \%$ schlechteren Verlaufs pro 30-min-Zeitverzögerung, sodass der Notwendigkeit eines zentralvenösen Zugangs oder eines Blasenkatheters im Vorfeld oder bei der
Narkoseeinleitung geklärt sein sollte. Bei eindeutiger Indikation eines zentralen Venenkatheters (ZVK) ist durchaus auch in Erwägung zu ziehen, dass der Interventionalist diesen in der Leiste legt. Bei Eingriffen mit absehbar längerer Eingriffszeit sollte ein Blasenkatheter gelegt werden.

Die Positionierung des Kopfes in einer sogenannten Nullposition ist während des Eingriffs wichtig, damit die Gefäßanatomie über den Zeitverlauf für den Interventionalisten reproduzierbar ist und damit 3D-Untersuchungen im Verlauf der Behandlung als Referenz herangezogen werden können. Hierfür ist unerlässlich, dass die Beatmungsschläuche und -kabel von Beginn an so positioniert werden, dass ein freies Bewegen der Röntgenröhren zirkulär um den Kopf des Patienten herum möglich ist. Bei Untersuchungen der Wirbelsäule ist das Management entsprechend anzupassen. Empfehlenswert sind Kabelverläufe für Blutdruck, Messungen des intrakranialen Drucks „„,intracranial pressure“, ICP) und der pulsoxymetrisch ge- messenen Sauerstoffsättigung $\left(\mathrm{S}_{\mathrm{p}} \mathrm{O}_{2}\right)$ sowie EKG usw., die möglichst am Angiographietisch verlaufen und nicht zu Stolperfallen werden.

Die Narkoseeinleitung kann durch die oben beschriebenen Maßnahmen erheblich beschleunigt werden. Die ständige Kommunikation zwischen Interventions- und Anästhesieteam erlaubt ein koordiniertes Arbeiten während des gesamten Eingriffs, ohne dass das Anästhesieteam unmittelbar am Patienten positioniert sein muss. Mehrere Überwachungsmonitore im Vor- und Untersuchungsraum sowie ein für den Neurointerventionalisten einsehbarer Monitor erlauben, dass Blutdruckschwankungen oder Herzrhythmusstörungen, die möglicherweise interventionsbedingt sein können, entsprechend kommentiert werden können. Im Einzfall kann es im Rahmen der Vasospasmusbehandlung mit Vasodilatanzien zu erheblichen Blutdruckabfällen kommen, denen mit Katecholamingaben begegnet werden muss. Generell sollte vor jeder Behandlung ein Zielblutdruckwert mit Grenzen abgesprochen 

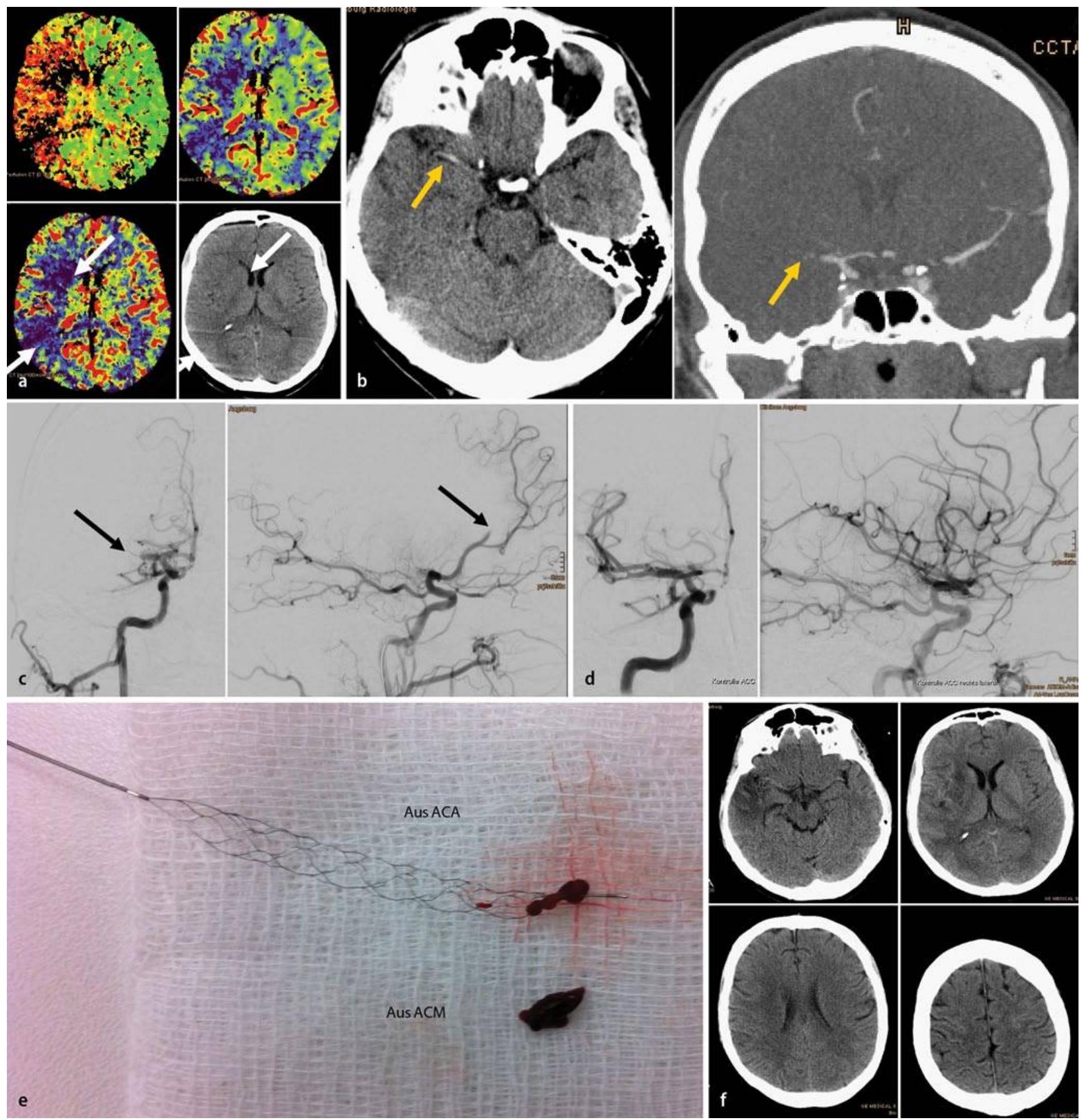

Abb. $2 \Delta$ Ein 53-jähriger Patient mit akutem Schlaganfall. a Im Computertomogramm (CT) und Perfusions-CT Infarktfrühzeichen mit definitivem rechtstemporalem Infarkt und Kaudatumkopf (weiße Pfeile) sowie drohendem Infarkt, der das gesamte Mediastromgebiet betrifft (rot Areal mit verzögerter Kontrastmittelanflutung, links oben). b Im nativen CT dichtes Mediazeichen (",dens artery sign“, links) durch intravasalen Thrombus und entsprechender Gefäßabbruch in der CT-Angiographie (rechts, gelbe Pfeile). c In der Angiographie zeigten sich eine höchstgradige Karotisstenose, die endovaskulär mit Stent versorgt wurde, sowie ein Verschluss der mittleren und vorderen Hirnarterie (Pfeile). d Nach Stent retrieving Rekanalisation. e Stent retriever und Nachweis der Blutgerinnsel, die aus der vorderen $(A C A)$ und der mittleren $(M C A)$ Hirnarterie entfernt wurden. f Schädel-CT-Kontrolle nach 14 Tagen mit nur kleinen umschriebenen Infarkten

werden. Andererseits kann beim Ziehen von Mikrokathetern aus der hinteren Zirkulation, v. a. beim Entfernen von Mikrokathetern nach AVM-Embolisationen, durch Zug auf den Hirnstamm oder bei Druck auf den Karotisbulbus eine Bradykardie bis zum Herzstillstand ausgelöst werden. Falls antizipiert, kann dieser
Komplikation bereits vor dem Ereignis oder perakut mit Atropin und ggf. Adrenalin - vorgebeugt werden. 


\section{Strahlenschutz}

Die Möglichkeit, aus verschiedenen Positionen innerhalb und außerhalb des Untersuchungsraums den Patienten zu überwachen, sollte auch aus Strahlenschutzgründen vom Anästhesieteam in Anspruch genommen werden. Eine zusätzlich zwischen Röntgenröhre und Anästhesist aufgestellte Bleiwand schützt das Anästhesiepersonal. Aus Strahlenschutzgründen befindet sich die Röntgenröhre im frontalen (Posterior-anterior, p.-a.)Strahlengang unterhalb des Patienten und im seitlichen Strahlengang auf der gegenüberliegenden Seite zum Interventionalisten. Damit ist die seitliche Röhre in unmittelbarer Nähe zum Anästhesiepersonal, das sich entsprechend durch Bleischürze, Schilddrüsenschutz und, wenn möglich, zusätzliche Bleiwände schützen muss. Der Abstand zur Röntgenröhre sollte möglichst groß sein, sodass es durchaus empfehlenswert ist, dass die Überwachung, soweit möglich, aus dem Schaltraum heraus erfolgt. Anastasian et al. [2] berichten über eine mittlere Strahlendosis im Gesicht eines Anästhesisten bei Neurointerventionen von $6,5 \pm 5,4 \mu \mathrm{Sv}$. Diese Dosis ist 6-mal höher als bei normalen angioraphischen Prozeduren und 3-mal höher als die Dosis, die der Interventionalist erhält. Dies begründet, wieso am Klinikum Augsburg seit vielen Jahren entsprechende Strahlenschutzvorrichtungen installiert werden. Hierzu zählt auch, dass jedwede Kontakte zum Patienten vorher angekündigt werden müssen. Zum einen, um Bewegungsartefakte zu vermeiden, zum anderen, um das Personal vor Strahlenexposition zu bewahren. Die bei neuen Angiographieanlagen zum Einsatz kommenden Niedrigdosisprogramme sind eine weitere Strahlenschutzmaßnahme, die aber nur dann wirksam ist, wenn sich das Personal an die Abstandsregeln und das Fernhalten vom aktiven Strahlengang hält.

\section{Angiographietisch}

Länger dauernde Interventionen gehen mit Patientenauskühlung einher. Dieser kann entgegengewirkt werden, in dem auf Körpertemperatur vorgewärmte Spülflüssigkeiten und Kontrastmittel appliziert werden. In den Tisch integrierte Wärmematten verhindert zudem das Auskühlen, v. a. von Kindern.

Die Tischkippung mit Kopfhoch- und Kopftieflagerung hat den Vorteil, dass dies sowohl bei Patienten mit erhöhtem ICP, aber auch zur ZVK-Anlage genutzt werden kann. Therapeutische Maßnahmen an Patienten mit erhöhtem ICP sind selten und betreffen in der Regel Patienten mit SAB und Vasospasmen, die kopfhoch gelagert werden können.

\section{Komplikationsmanagement}

Dem Komplikationsmanagement (- Tab.3) kommt intraprozedural ein besonders hoher Stellenwert zu. Spinale Komplikationen sind selten und führen bei thrombembolischem Verschluss der Spinalarterie zur Querschnittslähmung. Intrakranielle Komplikationen sind mit $0,3 \%$ bei diagnostischen und bis zu $10 \%$ bei therapeutischen Eingriffen häufiger und können in weniger als $1 \%$ tödlich verlaufen oder zu anhaltenden neurologischen Ausfällen führen.

Beim Sondieren von Hirngefäßen, insbesondere von Aneurysmen, ist Relaxierung angezeigt, um Gefäßperforationen zu vermeiden. Bei endovaskulären Eingriffen gehen Blutungen häufig mit schlechten Ergebnissen einher, insbesondere wenn Gefäße oder Aneurysmen unter Antiaggregationsbehandlung rupturieren. Davon trennen muss man eine durch Mikrodraht induzierte Dissektion mit in der Regel umschriebenen benignen Blutungen. Bei anhaltenden Blutungen muss versucht werden, die Blutungsstelle zu verschließen. Dies kann durch passageren Gefäßverschluss mithilfe eines Ballons oder durch Platinspiralen bis hin zum Gebrauch von Flüssigembolisaten reichen. Ziel sollte sein, das gehirnversorgende Gefäß zu erhalten.

\section{Antikoagulation und Antiaggregation}

Die häufigsten Komplikationen sind mit nahezu 3\% Thromboembolien. Um diese zu vermeiden, sollten die Kathetersysteme kontinuierlich gespült und eine Heparinisierung ( $\bullet$ Tab. 2) angestrebt werden. Bei hohem prozeduralem Blutungsrisi- ko wie frisch rupturiertem Aneurysma muss der Interventionalist in einer Risikoabwägung Vor- und Nachteile der Heparinisierung beurteilen. Bis heute gibt es keine Standardprozedur für die periprozedurale Antikoagulation. Bei Wahleingriffen erfolgt die Heparinisierung per i.v.-Bolus oder körpergewichtsadaptiert über eine Spritzenpumpe. Eine Kontrolle kann im Verlauf durch Messungen der "activated clotting time" (ACT) erfolgen. Bei Neurointerventionen wird dabei eine Verdopplung der ACT angestrebt.

Patienten mit SAB haben ein höheres Risiko für eine Hyperkoagulabilität, sodass die Heparinisierung häufig nach Platzierung der ersten Platinspirale erfolgt, weil danach das Rupturrisiko deutlich geringer ist als zu Beginn der Intervention. Zur Vermeidung von Thrombosen wird bei geplantem Einsatz von Stents eine Prämedikation mit doppelter Thrombozytenaggregation durchgeführt, die 3 bis 5 Tage vorher beginnt und nach Stenting für einen bestimmten Zeitraum fortgeführt wird. Das Augsburger Schema ist in Tab. 4 dargestellt. Auch hier konnte bislang kein Konsens gefunden werden, sodass individuelle Erfahrungen in die Therapieentscheidung einfließen.

Bei Auftreten frischer Thromben während des Eingriffs kann je nach Thrombusmasse und abhängig davon, ob ein Gefäß verschlossen ist oder ein umspülter Thrombus vorliegt, die Therapie entsprechend eskaliert werden. Zunächst können der systolische Blutdruck angehoben und 300-500 mg Aspirin i.v. appliziert werden. Zeitgleich sollte entweder mithilfe der ACT-Messung oder aus Erfahrung beurteilt werden, ob eine ausreichende Heparinisierung vorliegt. Aus jahrelanger Erfahrung mit ACT-Messungen kann davon ausgegangen werden, dass bei einem 70-90 kg schweren Patienten mit 5000 IE Heparin als Bolus nach ca. 1,5-3 h eine erneute Heparinisierung mit 20003000 IE erforderlich ist. Wirkt die Thrombusmasse okklusiv, sollte eine i.v.-GPIIa/ IIIb-Behandlung durchgeführt werden. Häufig zum Einsatz kommen Aggrastat, ReoPro ${ }^{\circ}$ oder Integrilin ${ }^{\circ}$. Diese Substanzen sind wirksamer als Fibrinolytika, weil es sich in der Regel um Thrombozytenaggregate handelt. Fibrinolytika bergen insbesondere in der Behandlung rupturierter 
Aneurysmen die Gefahr, dass es zu Rerupturen mit fatalen Blutungen kommt, die bei Verwendung von GP-IIa/IIB-Hemmern deutlich seltener zu sein scheinen. Ebenso sollten aus diesem Grund Eingriffe, wie die Anlage einer externen Ventrikeldrainage bei Patienten mit SAB, vor der endovaskulären Versorgung eines Aneurysmas erfolgen.

Während sich der Umgang mit Vitamin-K-Antagonisten im periprozeduralen Antikoagulationsmanagement über Jahrzehnte etabliert hat, kommen heute neue orale Antikoagulanzien zur Anwendung, die bislang selten eingesetzt werden und deren Management noch nicht routiniert abläuft. Es handelt sich um direkte Antagonisten der Gerinnungsfaktoren FXa (Rivaroxaban und Apixaban) und Thrombin (Dabigatranetexilat), für die es kein spezifisches Antidot gibt. Zum Einsatz dieser Medikamente bei neurointerventionellen Maßnahmen liegen keine Stellungnahmen oder Empfehlungen vor. Nach Sauer et al. [39] scheint die Blutungskomplikation in Form einer Leistenarterienpunktion bei Herzkatheteruntersuchungen durch lokale Kompression beherrschbar, während spinale oder epidurale Blutungen keiner Maßnahme zugänglich sind und eine dauerhafte Paraplegie hervorrufen können. Die Indikation zur Durchführung eines neurointerventionellen Eingriffs ist demnach unmittelbar von der Dringlichkeit und der drohenden Gefährdung des Patienten durch Unterlassen abhängig. Aus diesem Grund sollte bei Notwendigkeit einer Antikoagulationsbehandlung insbesondere in Kombination mit der Gabe von Thrombozytenaggregationshemmern eher auf Marcumar umgestellt werden, da hier langjährige Erfahrungen vorliegen.

\section{Intraprozedurale Volumen-Com- putertomographie-Untersuchung}

Moderne Angiographieanlagen bieten die Möglichkeit der intra- und postprozeduralen Volumen-CT-Untersuchung, sodass der Patient bei ausgedehnten Blutungen direkt zur Blutungsevakuation in den OP verbracht werden kann. Dies erspart unnötige Umlagerungsprozeduren und v. a. für den Patienten relevante Zeit, bis eine raumforderne Blutung entfernt wird. Falls die Blutungen keine unmittelbare Relevanz haben, kann die Intervention fortgeführt und abschließend erneut diskutiert werden, wie das weitere therapeutische Prozedere aussieht.

\section{Spezielle Interventionen}

Im Folgenden wird anhand von zwei häufigen und relevanten Erkrankungen auf aktuelle und spezifische Indikationen und Probleme hingewiesen. Hierbei handelt es sich um Aneurysmen (• Abb. 1) und akute Rekanalisationsbehandlungen (• Abb. 2), die im Augsburger Patientenkollektiv fast zwei Drittel der knapp 300 zerebralen und spinalen Gefäßeingriffe im Jahr ausmachen.

\section{Endovaskuläre \\ Aneurysmabehandlung}

Das Clipping eines Aneurysmas erfolgt unter direkter Sicht, sodass es komplett ausgeschaltet werden kann. Dieses Argument ist nach wie vor Bestandteil der neurochirurgischen Aufklärung, obwohl bei Kontrollangiographien nach Clipping in 5-9\% der Fälle Aneurysmareste erkannt werden. Die Langzeitergebnisse der ISAT [29] zeigten keine signifikanten Unterschiede zwischen operativem Clipping und endovaskulärer Behandlung. Dies war für viele überraschend, weil sich die Ergebnisse in der Einjahreskontrolle signifikant unterschieden und zum Studienabbruch geführt hatten. In dem endovaskulär behandelten Kollektiv fand sich ein schlechtes Ergebnis oder Tod in 23,7 vs. 30,6\% in dem mit Clip versorgten Kollektiv [48]. Dieser signifikante Unterschied ist in der Fünfjahresverlaufskontrolle nicht mehr nachweisbar.

\section{Clipping vs. Coiling}

In Subgruppenanalysen lassen sich im Verlauf noch signifikante Unterschiede nachweisen. Diese betreffen die postprozedurale Epilepsie und kognitive Funktionsstörungen, die nach offener Operation häufiger auftreten [43]. Eindeutige Argumente für die endovaskuläre Behandlung eines Aneurysmas nach Blutung wurden zuletzt durch die Barrow Ruptered Aneurysm Trial (BRAT, [27]) untermauert. Bei 471 behandelten Patien- ten lag nach einem Jahr ein $\mathrm{mRS}>2$ bei $33,7 \%$ in der mit Clip operierten Gruppe und bei 23,2\% in der mit Coils behandelten Gruppe vor $(\mathrm{p}=0,02)$.

Risselada et al. [38] haben die Prädiktoren der 60-Tage-Sterblichkeit im Rahmen der ISAT analysiert und gefunden, dass Patientenalter, Aneurysmagröße und Blutungsschwere [Fisher- und World-Federation-of-Neurological-Surgeons(WFNS)Grad] die Letalität beeinflussen, während das Geschlecht des Patienten sowie Lage und Zahl der Aneurysmen keinen Einfluss haben. Aus diesen Daten könnte man schlussfolgern, dass bei Detektion mehrerer Aneurysmen nur das Rupturierte der unmittelbaren Behandlung bedarf und die weiteren, bei bekannt höheren Blutungsrisiken, zu einem späteren Zeitpunkt versorgt werden können. Diese Vorgehensweise muss individuell und interdisziplinär entschieden werden, damit bei rupturierten Aneurysmen im Fall von Vasospasmen trotz der nichtbehandelten Zweitaneurysmen eine medikamentöse Vasospasmustherapie begonnen werden kann.

In der Studie Cerebral Aneurysm Rerupture After Treatment (CARAT, [14]) wurden Häufigkeit und Auswirkungen intraprozeduraler Rupturen bei der Behandlung von rupturierten Aneurysmen untersucht. Beim Clipping traten in 19\% der Fälle intraprozedurale Blutungen auf, beim Coiling nur in 5\% der Fälle. Die mit den Blutungen verbundenen Risiken Tod und schlechter klinischer Verlauf sind bei $31 \%$ der operierten, aber bei $63 \%$ der endovaskulär versorgten Patienten aufgetreten. Oberste Priorität v. a. beim endovaskulären Zugang hat demnach die Vermeidung einer intraprozeduralen Aneurysmaruptur.

\section{Clipping oder Coiling in Deutschland}

Die ISAT war eine Zäsur in der Behandlung der rupturierten Aneurysmen hin zur endovaskulären Versorgung. Dies wird auch durch die diskutierten Langzeitergebnisse unterstützt. Nichtsdestotrotz hat diese Zäsur sich nicht auf die Aneurymen beschränkt, die im Rahmen der ISAT behandelt wurden, sondern wurde auf alle Aneurysmen, ob inzidentell oder geblutet, erweitert. Diese Auswirkung spiegelt sich im Qualitätsbericht 2008 der Allgemei- 
nen Ortskrankenkasse (AOK), basierend auf den Qualitätsberichten der einzelnen Kliniken, wider: In der Gesamtzahl wurden zu diesem Zeitpunkt in Deutschland 3823 Aneurysmen gecoilt (53\%) und 3390 geclippt (47\%). Diese nahezu gleiche Verteilung beruht darauf, dass nicht an jeder neurochirurgischen Klinik auch die Möglichkeit einer endovaskulären Behandlung besteht. Aufgrunddessen wurde 2010 eine Umfrage des Berufsverbands der Deutschen Neuroradiologen (BDNR) durchgeführt. Es haben sich 45 der 86 in Deutschland neurointerventionell aktiven Kliniken, die zusammen mit einer Neurochirurgie an einem Standort tätig sind, an der Umfrage beteiligt. In der Gesamtheit wurden 62\% der Aneurysmen endovaskulär und $38 \%$ operativ versorgt. Betrachtet man die High-volume-Zentren, die mehr als 50 Aneurysmen im Jahr behandeln, zeigt sich, dass an Häusern in kommunaler oder privater Trägerschaft im Mittel 76\% der Fälle endovaskulär und $24 \%$ operativ sowie an Universitätskliniken 64\% der Fälle endovaskulär und 36\% operativ versorgt werden. Dieser Unterschied wird von den Universitätskliniken in erster Linie mit der Weiterbildungsverpflichtung begründet.

\section{Innovationen}

Ein wichtiger, nicht zu vernachlässigender Punkt ist der Innovationsschub, den die endovaskuläre Methode innerhalb der letzten Jahre erfahren hat. Während die operative Methode von der intraoperativen Fluoreszenzangiographie, der Möglichkeit zu navigieren und dem intraoperativen Monitoring profitieren konnte, waren die Innovationen aufseiten der endovaskulären Behandlung durch die Weiterentwicklung der Platinspiralen bestimmt, die durch unterschiedliche Konfigurationen und Weichheitsgrade ein dichteres Ausfüllen der Aneurysmen erlaubt und damit die Rezidiv- sowie die Nachbehandlungsrate senkt. Hinzu kommen additive Methoden wie das Remodeling mithilfe eines Ballons, das Stent-unterstützte Coiling und die Verwendung von in das Trägergefäß oder in das Aneurysma eingebrachten Flow diverters.

\section{Remodeling und Stent-unterstütztes Coiling von Aneurysmen}

Beim Remodeling wird ein Ballon an der Aneurysmabasis platziert und dieser passager mit einem Gemisch aus Wasser und Kontrastmittel gefüllt, während über einen zweiten Mikrokatheter das Aneurysma mit Coils behandelt wird. Dies erlaubt einen besseren Abschluss der Spiralen gegenüber dem Trägergefäß und ein dichteres Ausfüllen des Aneurysmasacks. Das Stent-unterstützte Coiling ist für breitbasige Aneurysmen vorgesehen, bei denen die Gefahr besteht, dass Coils in das Trägergefäß vorfallen.

\section{„Flow diverter"}

In jüngster Zeit wurden Flussbegradiger (Flow diverter; - Abb. 1) in die Aneurysmabehandlung eingeführt. Über die Indikation für deren Einsatz ist noch nicht entschieden. In Europa gibt es aktuell keine Anwendungseinschränkungen, während sich der Einsatz in den USA an der PUFS-Studie orientiert, die die Food and Drug Administration (FDA) zur Zulassung herangezogen hat, und in der große und Riesenaneurysmen mit breitem Hals behandelt wurden. Entsprechend bezieht sich die Einschränkung auf Erwachsene, die älter als 21 Jahre sind und deren Aneurysmen in der ACI zwischen dem petrösen und oberen hypophysalen Segment liegen. Die Ergebnisse der PUFS-Studie wurden mit Literaturergebnissen verglichen, und es zeigte sich, dass ein kompletter Verschluss nach 180 Tagen in $82 \%$ der Fälle und nach einem Jahr in $86 \%$ gegeben war. Die Komplikationsrate schwerer Schlaganfall oder Tod wurde mit 5,6\% angegeben.

\section{Beschichtete Platinspiralen}

In den vergangenen 10 Jahren wurden beschichtete Platinspiralen auf den Markt gebracht, die mit Vorteilen im Behandlungsergebnis und geringeren Nachbehandlungsraten einhergehen sollten. In der Studie Hydrogel-coated Coils versus Bare Platinum Coils for the Endovascular Treatment of Intracranial Aneurysms (HELPS, [47]) wurden hydrogelbeschichtete mit regulären Platinspiralen verglichen. Hier konnte gezeigt werden, dass die Nachbehandlungsrate mit 3,5\% sehr gering ist und deutlich unter den Literaturangaben von 5-20\% liegt. Die Nachbehandlungsrate betrug bei Verwendung von HydroCoils 2,9\% und bei regulären Coils 3,6\%. Die Packungsdichte war aufgrund der schwellenden Hydrogelbeschichtung deutlich höher als die der regulären Platinspiralen (68,3 vs. $24,9 \%$ ). Aktuell werden Patienten mit inzidentellen und rupturierten Aneurysmen in eine weitere Studie (GREAT) mit Verwendung von HydroSoft-Coils oder Platinspiralen aufgenommen.

In der Cerecyte-Studie [11] wurden Platinspiralen, die einen innerhalb der Spirale verlaufenden resorbierbaren Faden enthalten, mit regulären Platinspiralen verglichen. Es fand sich kein signifikanter Unterschied im Verlauf zwischen beiden Gruppen bei den rupturierten Aneurysmen (96\% leben und sind unabhängig) und den nichtrupturierten Aneurysmen. Insgesamt waren die prozeduralen Ereignisse in der Cerecyte-Gruppe (15\%, 36 von 247 Patienten) häufiger als in der Platinspiralgruppe $(10 \%, 25$ von 250 Patienten). Nichtsdestotrotz sind die klinischen Verläufe (rupturiert und inzidentell) besser als in bislang publizierten randomisierten Studien wie der bereits erwähnten ISAT oder der International Study of Unruptured Intracranial Aneurysms (ISUIA).

In die Matrix and Platinum Science (MAPS) Trial wurden 626 Patienten mit rupturierten (36\%) und inzidentellen (64\%) Aneurysmen für die Behandlung mit Platinspiralen oder mit resorbierbarem Material ummanteleten Platinspiralen randomisiert (Daten unveröffentlicht). Die Studie wurde in $43 \mathrm{Kli}$ niken in 11 Ländern durchgeführt. Die Ergebnisse der Studie zeigen, dass beschichtete Matrix-Coils tendenziell, aber nicht signifikant besser als reguläre Spiralen sind. In der Gruppe mit inzidentellen Aneurysmen wiesen 96\% der Patienten nach 15 Monaten einen $m R S \leq 2$ auf. Dieser ist deutlich besser als der zu erwartende von $90 \%$ (nach ISUIA). Ebenso verhält es sich mit den rupturierten Aneurysmen, bei denen bei $90 \%$ der Patienten nach 15 Monaten ein $\mathrm{mRS} \leq 2$ bestimmt wurde. Nach der ISAT wären lediglich $76 \% \mathrm{zu}$ erwarten. Aus dieser Studie lässt sich sehr gut ableiten, dass sich die endovaskulä- 
re Therapie aufgrund höherer Fallzahlen und geringerem Einfluss von Lernkurven deutlich im Bezug auf klinischen Verlauf und Nachbehandlungsrate verbessert hat. Aus der MAPS Trial lassen sich aber noch weitere Schlussfolgerungen ableiten. Ein initial komplett oder subtotal verschlossenes Aneurysma ist ein Prädiktor für fehlende Rekanalisation, und Aneurysmen mit Stent und Coils haben ebenfalls geringere Nachbehandlungsraten.

\section{Schlaganfall}

Die LIF ist mittlerweile ein etabliertes Verfahren zur Behandlung proximaler Hirngefäßverschlüsse. Die Erweiterung des Therapiespektrums um mechanische Verfahren hat dazu geführt, dass die Behandlungszahlen in den letzten Jahren rasant zugenommen haben. Die sich daraus entwickelnden Versorgungsansprüche sind mit speziellen Anforderungen an die Verfügbarkeit von Räumlichkeiten sowie ärztlichem, technischem und pflegerischem Personal verknüpft, die rund um die Uhr notfallmäßig zu Verfügung stehen müssen.

\section{Multimodales \\ Behandlungskonzept}

Zeumer et al. [51] leisteten seit den 1980er Jahren Pionierarbeit in Bezug auf die LIF der Hirngefäße und Lyseschemata bis hin zum „Bridging“-Therapieansatz, der Kombination aus systemischer und lokaler Lyse. Bereits in der Studie Prolyse in Acute Cerebral Thromboembolism (PROACT) II konnte gezeigt werden, dass die intraarterielle Thrombolyse mit Prourokinase innerhalb von $6 \mathrm{~h}$ sicher sowie wirksam ist und zu einer signifikant höheren Rate funktionell unabhängiger Patienten führte, auch bei initial schwerer Schlaganfallsysmptomatik und proximalem Gefäßverschluss. In jüngster Zeit sicherlich wegweisend für die Akzeptanz des Verfahrens war die von Mattle et al. [26] publizierte Arbeit über Patienten mit im CT gesicherten Mediahauptstammverschluss und eindeutigem Vorteil der Urokinase-LIF gegenüber der intravenösen Thrombolyse (IVT). Ein guter klinischer Verlauf mit mRs $\leq 2$ fand sich bei nahezu 30\% mehr LIF- als bei IVT-Patienten. Hinzu kommt, dass bei einer Thrombuslänge von $8 \mathrm{~mm}$ im Mediahauptstamm keine Rekanalisation durch IVT zu erwarten ist [37], sodass bei entsprechender Thrombuslast folgerichtig die intraarterielle Behandlung infrage kommt.

Am Klinikum Augsburg wird, wenn es der zeitliche Verlauf zulässt, regelhaft eine Bridging-Behandlung initiiert, sodass innerhalb von 4,5 h mit IVT begonnen wird, die dann intraarteriell fortgesetzt wird. Befundabhängig erfolgt die intraarterielle Rekanalisation entweder mit intraarterieller medikamentöser Lyse oder mechanisch. Falls bei der mechanischen Rekanalisation Thrombusverlagerungen in die Peripherie oder in ein anderes größeres Gefäß auftreten, kann mit dem residuell zu Verfügung stehenden Fibrinolytikum weiter lokal behandelt werden. Die einzelnen Behandlungsoptionen münden in ein multimodales Konzept, das unterschiedliche Applikationen von Fibrinolytika und unterschiedliche mechanische Rekanalisationsverfahren (Saugen, Stent retrievers bis hin zu PTA und Stenting, $[4,5,13,17$, $25,40,44,46])$ umfasst. Vorteil der mechanischen Verfahren ist, dass diese in Bezug auf Rekanalisation am wirksamsten sind [36]. Hinzu kommt, dass diese Systeme bei Patienten mit Kontraindikationen für Fibrinolytika eingesetzt werden können.

\section{Thrombektomiesysteme wie Stent retriever und Absaugsysteme}

Aktuell werden nahezu 10 verschiedene Stent retriever angeboten; derzeit werden Systeme wie Solitaire ${ }^{\varpi}$ der Fa. ev3covidien und Trevo ${ }^{\star}$ der Fa. Stryker am häufigsten benutzt. Bei den Stent retrievern handelt es sich um selbstexpandierbare Stents, die innerhalb des Thrombus platziert werden, dort für wenige Minuten verweilen und anschließend mit dem Ziel, den Thrombus herauszuziehen, entfernt werden (• Abb. 2). Weitere häufig eingesetzte Systeme sind das MERCI ${ }^{\circledR}$-System der Fa. Stryker/Concentric, das wie ein Korkenzieher im entfalteten Zustand aussieht und mit dem ebenfalls das Blutgerinnsel herausgezogen werden soll. Das Absaugsystem der Fa. Penumbra hat sich ebenfalls durch hohe Rekanalisationsraten ausgezeichnet.
Nach wie vor ist die Rekanalisationsrate der wichtigste Faktor für einen guten klinischen Verlauf. Rha u. Saver [36] konnten in einer Metaanalyse von 33 Studien mit 998 Patienten zeigen, dass ein guter funktioneller klinischer Verlauf nach 3 Monaten häufiger bei Patienten mit Rekanalisation ist als bei erfolgloser Rekanalisation mit persistierendem Verschluss ["odds ratio" 4,43, 95\%-Konfidenzintervall (95\%-KI) 3,32-5,91]. Kritisch anzumerken bleibt, dass die zuletzt publizierten Ergebnisse zwar mit hohen Rekanalisationsraten aufwarten konnten, diese aber sich nicht in guten klinischen Verläufen widerspiegelten. In der initialen Penumbra-Studie [5] betrug die Rekanalisationsrate $100 \%$. Trotzdem war nur bei $25 \%$ der Patienten ein guter klinischer Verlauf zu verzeichnen. Eine mögliche Ursache für diese schlechten Ergebnisse mag in der Zeitverzögerung von 2,2 h vom Eintreffen des Patienten $(1,9 \mathrm{~h}$ ) bis zur Gefäßpunktion $(4,1 \mathrm{~h})$ liegen.

Ähnliche Ergebnisse wurden in einer von Taschner et al. [46] publizierten Arbeit beschrieben. Hier wurden Daten der Rekanalisationsbehandlung ohne und mit Penumbra-System verglichen. Ähnlich wie in der Penumbra-Studie waren die Rekanalisationsergebnisse mit 78 vs. $53 \%$ beeindruckend. Allerdings war wie in der Studie der klinische Verlauf mit $m R S \leq 2$ in der Penumbra-Gruppe bei $15 \%$ der Teilnehmer sehr schlecht und in der Kontrollgruppe trotz geringerer Rekanalisationsrate mit 35\% deutlich besser.

Diese Ergebnisse sind ernüchternd und sprechen nicht für eine erfolgreiche zukunftweisende Methode. Dies wird auch durch die im Mai 2012 ergangene Mitteilung, dass in den USA die Studie Interventional Management of Stroke (IMS) III abgebrochen wurde, bestärkt. In dieser Studie erfolgte eine BridgingBehandlung unter Verwendung mechanischer Systeme; hierbei schnitt die endovaskuläre Gruppe mit 40,8\%igem gutem Verlauf nicht signifikant unterschiedlich zur IVT-Gruppe mit 38,7\%igem gutem Verlauf ab [7]. Ursachen liegen möglicherweise darin begründet, dass bei 434 endovaskulär behandelten Patienten aufgrund der Zulassungen überwiegend die Systeme Merci, Penumbra und Ekos und in lediglich 5 Fällen Stent retriever ein- 
gesetzt wurden. In der Studie Mechanical Retrieval and Recanalization of Stroke Clots Using Embolectomy (MR RESCUE) konnte bei 118 Patienten, die zwischen 2004 und 2011 aufgenommen wurden, ebenfalls kein Vorteil der mechanischen Systeme Merci und Penumbra gegenüber der Standardtherapie mit IVT gezeigt werden [24]. Diese Systeme können offensichtlich durch hohe Rekanalisationsraten, aber nicht durch gute klinische Ergebnisse überzeugen. In der Studie Synthesis Expansion: A Randomized Controlled Trial on Intra-Arterial Versus Intravenous Thrombolysis in Acute Ischemic Stroke (SYNTHESIS) konnte ebenfalls nicht über einen Vorteil der endovaskulären Behandlung gegenüber der IVT berichtet werden [10]. In dieser Studie wurden 163 Patienten endovaskulär behandelt; hierbei wurden in 37 Fällen mechanische Systeme wie Stent retriever $(n=23)$, Penumbra $(n=9)$ oder Mer$\mathrm{ci}(\mathrm{n}=5)$ eingesetzt. Ansonsten wurde das Blutgerinnsel durch Mikrodrahtmanipulationen und im Mittel mithilfe der Gabe von $40 \mathrm{mg}$ rtPA beseitigt.

Den im Februar 2013 publizierten Studien IMS III, SYNTHESIS und MR RESCUE ist gemeinsam, dass die neueste Generation an mechanischen Rekanalisationssystemen unterrepräsentiert ist. Die Ergebnisse beim Einsatz von Stent retriever zeigen, dass mit diesen Systemen eine erheblich schnellere Rekanalisation, und zwar binnen $60 \mathrm{~min}$, möglich ist [25], die sich zudem in guten klinischen Verläufen widerspiegelt $[13,40]$. Dass die Stent retriever den älteren Systemen überlegen sind, konnte in den randomisierten Studien Thrombectomy Revascularization of Large Vessel Occlusions in Acute Ischemic Stroke (TREVO) 2 und Solitaire $^{\mathrm{Ts}}$ FR With the Intention For Thrombectomy (SWIFT) nachgewiesen werden [33]. In diesen Studien wurden die Stent retriever Trevo ${ }^{\circledast}$ und Solitaire ${ }^{\text {Tw }}$ mit dem Merci-System verglichen [33]. Beim Vergleich Merci $^{\circledast}$ mit Trevo ${ }^{\circledast}$ waren die Trevo-Ergebnisse deutlich besser, bezogen auf die Gefäßrekanalisation mit 68 vs. $44 \%$. Einen guten klinischen Verlauf (90 Tage mRs 0-2) zeigten $40 \%$ der mit Trevo $^{\circledR}$ und $21,8 \%$ der mit Merci ${ }^{\circledR}$ behandelten Patienten. Diese Ergebnisse wurden in der SWIFT-Vergleichsstudie zwi- schen Solitaire ${ }^{\circledast}$ und Merci ${ }^{\varpi}$ mit gutem klinischen Verlauf nach 90 Tagen mit 58 vs. $33 \%$ bestätigt und waren für das Steering Committee Anlass, die Studie vorzeitig abzubrechen.

Was ist für diese guten Ergebnisse ursächlich? Zum einen, dass sich hier etablierte Schlaganfallkliniken mit hohen Fallzahlen zusammengeschlossen haben, die über die entsprechende Erfahrung in der Patientenselektion verfügen. Zum anderen bewirkt die Entfaltung des Stents eine kürzer oder länger währende Rekanalisation, sodass die Durchblutung passager wiederhergestellt ist, da das Thrombusmaterial zunächst zwischen Stent-Streben und Gefäßwand verlagert wird. Nach wenigen Minuten dringt das Thrombusmaterial in der Regel wieder in den Stent ein, verhakt sich zwischen

\begin{tabular}{|c|c|}
\hline \multicolumn{2}{|c|}{ Abkürzungen } \\
\hline$A C l$ & A. carotis interna \\
\hline ACT & "activated clotting time" \\
\hline ASS & Acetylsalicylsäure \\
\hline AVF & arteriovenöse Fistel \\
\hline AVM & arteriovenöse Malformation \\
\hline BRAT & Barrow Ruptered Aneurysm Trial \\
\hline CARAT & $\begin{array}{l}\text { Cerebral Aneurysm Rerupture } \\
\text { After Treatment }\end{array}$ \\
\hline$C T$ & Computertomogramm \\
\hline EKG & Elektrokardiogramm \\
\hline GREAT & $\begin{array}{l}\text { German Randomized Endovas- } \\
\text { cular Aneurysm Trial }\end{array}$ \\
\hline GP $/ l a / I I b$ & Glykoprotein Ilb/Illa \\
\hline HELPS & $\begin{array}{l}\text { Hydrogel-coated Coils versus } \\
\text { Bare Platinum Coils for the Endo- } \\
\text { vascular Treatment of Intracranial } \\
\text { Aneurysms }\end{array}$ \\
\hline ICP & $\begin{array}{l}\text { „intracranial pressure“ (intrakra- } \\
\text { nialer Druck) }\end{array}$ \\
\hline IMS & $\begin{array}{l}\text { Interventional Management of } \\
\text { Stroke }\end{array}$ \\
\hline INR & interventionelle Neuroradiologie \\
\hline ISAT & $\begin{array}{l}\text { International Subarachnoid An- } \\
\text { eurysm Trial }\end{array}$ \\
\hline ISUIA & $\begin{array}{l}\text { International Study of Unruptu- } \\
\text { red Intracranial Aneurysms }\end{array}$ \\
\hline ITN & Intubationsnarkose \\
\hline IVT & intravenöse Thrombolyse \\
\hline LIF & lokale intraarterielle Fibrinolyse \\
\hline$L M W$ & "low molecular weight" \\
\hline MAP & $\begin{array}{l}\text { "mean arterial pressure" (mittle- } \\
\text { rer arterieller Druck) }\end{array}$ \\
\hline MAPS & $\begin{array}{l}\text { Matrix and Platinum Science } \\
\text { (Trial) }\end{array}$ \\
\hline
\end{tabular}

den Stent-Streben und kann dann nach 3-5 min zusammen mit dem Stent-System retrahiert werden. Unter Aspiration wird dann der Stent retriever mit dem Blutgerinnsel durch den Führungskatheter entfernt.

\section{Patientenselektion}

Prädiktoren für einen guten klinischen Verlauf sind nach Galimanis et al. [17]: periphere Gefäßverschlüsse, Rekanalisation, Hypercholesterinämie, gute Kollateralen, geringer Wert in der National Institutes of Health Stroke Scale (NIHSS), jüngere Patienten, Fehlen eines Diabetes mellitus und keine vorherige antithrombotische Therapie. Der Zeitpunkt der Behandlung war nur dann ein Prädiktor für einen guten Verlauf, wenn die Gefäßkollateralen ausgeschlossen wurden. Zusam-

\begin{tabular}{|c|c|}
\hline $\begin{array}{l}\text { MRRES- } \\
\text { CUE }\end{array}$ & $\begin{array}{l}\text { Mechanical Retrieval and Reca- } \\
\text { nalization of Stroke Clots Using } \\
\text { Embolectomy }\end{array}$ \\
\hline$m R S$ & modified Rankin Score \\
\hline NIHSS & $\begin{array}{l}\text { National Institutes of Health } \\
\text { Stroke Scale }\end{array}$ \\
\hline p.-a. & posterior-anterior (Strahlengang) \\
\hline PROACT & $\begin{array}{l}\text { Prolyse in Acute Cerebral Throm- } \\
\text { boembolism }\end{array}$ \\
\hline PTA & $\begin{array}{l}\text { perkutane transluminale Angio- } \\
\text { plastie }\end{array}$ \\
\hline PTT & $\begin{array}{l}\text { "partial thromboplastin time" } \\
\text { (partielle Thromboplastinzeit) }\end{array}$ \\
\hline$r t-P A$ & $\begin{array}{l}\text { „recombinant tissue-type plas- } \\
\text { minogen activator" }\end{array}$ \\
\hline$S A B$ & Subarachnoidalblutung \\
\hline $\begin{array}{l}\text { SAMM- } \\
\text { PRIS }\end{array}$ & $\begin{array}{l}\text { Stenting and Aggressive Medical } \\
\text { Management for Preventing } \\
\text { Recurrent stroke in Intracranial } \\
\text { Stenosis }\end{array}$ \\
\hline $\mathrm{S}_{p} \mathrm{O}_{2}$ & $\begin{array}{l}\text { pulsoxymetrisch gemessene } \\
\text { Sauerstoffsättigung }\end{array}$ \\
\hline SWIFT & $\begin{array}{l}\text { Solitaire }{ }^{\mathrm{TM}} \text { FR With the Intention } \\
\text { For Thrombectomy }\end{array}$ \\
\hline $\begin{array}{l}\text { SYNTHE- } \\
\text { SIS }\end{array}$ & $\begin{array}{l}\text { Synthesis Expansion: A Randomi- } \\
\text { zed Controlled Trial on Intra-Arte- } \\
\text { rial Versus Intravenous Thrombo- } \\
\text { lysis in Acute Ischemic Stroke }\end{array}$ \\
\hline TREVO & $\begin{array}{l}\text { Thrombectomy Revasculariza- } \\
\text { tion of Large Vessel Occlusions in } \\
\text { Acute Ischemic Stroke }\end{array}$ \\
\hline WASID & $\begin{array}{l}\text { Warfarin-Aspirin Symptomatic } \\
\text { Intracranial Disease }\end{array}$ \\
\hline$Z V K$ & zentraler Venenkatheter \\
\hline
\end{tabular}


menfassend lässt sich konstatieren, dass der wichtigste Aspekt die Patientenselektion ist. Diese erfolgt durch die klinische Untersuchung und v. a. durch die initiale Bildgebung und ist therapientscheidend (• Abb. 2).

Bei sich bereits demarkierenden Infarkten muss analysiert werden, ob es eloquente Areale gibt, die erhaltungswürdig sind. Bei fehlendem intrakraniellem Verschlussnachweis sollte eine vorgeschaltete zervikale oder im Bereich der Schädelbasis lokalisierte Stenose oder ein Verschluss durch Arteriosklerose oder Dissektion in Betracht gezogen werden, die es möglicherweise aus hämodynamischen Gründen zu eröffnen gilt. Genauere Analysen der Kollateralen, insbesondere der leptomeningealen Kollateralen können in der Angiographie mithilfe des CT oder MRT erfolgen. Zusätzliche Perfusionsmessungen können insbesondere bei hämodynamisch bedingten Durchblutungsstörungen hilfreich sein. Aus den aufgeführten Punkten lässt sich erschließen, dass rigide Zeitfenster für eine Therapieentscheidung nicht ausreichen. Bei fehlender Kollateralversorgung ist im CT bereits nach weniger als $1 \mathrm{~h}$ ein demarkierter Infarkt zu erkennen, der weder systemisch noch intraarteriell behandelt werden sollte.

Die intraarterielle Rekanalisation wird in den kommenden Jahren nicht zuletzt wegen der demografischen Entwicklung immer häufiger werden. Aktuell werden ca. 85\% der Fläche der Bundesrepublik durch Zentren mit einem Einzugsbereich von $60 \mathrm{~km}$ abgedeckt. Die Therapieprozedure ist nicht allzu kompliziert und kann von Nichtneurointerventionalisten relativ rasch erlernt werden. Risiken ergeben sich aus der falschen Indikationsstellung zur Behandlung, die potenziell mit schlechten Verläufen assoziiert ist, aus inadäquatem Komplikationsmanagement und v. a. daraus, dass die Qualität der Ergebnisse eben nicht einfach aus dem Erfolg der Rekanalisation resultiert. Konsequenterweise sollten Patienten mit schwerem Schlaganfall aus einem peripheren Haus entsprechend der Arbeit von Pfefferkorn et al. [34] „Drip, ship, and retrieve ..." in ein neurovaskuläres Zentrum verbracht werden.
Fazit

- Die INR zeichnet sich durch hohe Innovationsbereitschaft bei der Behandlung von Gefäßerkrankungen des Zentralnervensystems aus. Der Schlaganfall mit Hirngefäßverschluss und die Hirnaneurysmen stehen dabei im Mittelpunkt.

- Die aktuell zunehmenden Behandlungszahlen haben nicht zu einer Vereinheitlichung der Therapie und der Abläufe geführt, sondern werden ständig und in immer kürzeren Abständen weiterentwickelt und modifiziert.

- Sowohl Behandlungsmethoden als auch die Rolle der Anästhesie, insbesondere beim Schlaganfall mit der Frage der ITN oder Analgosedierung, können nur in Kenntnis der aktuellen Fakten, Fragestellungen und Zusammenhänge diskutiert werden.

\section{Korrespondenzadresse}

\section{Prof. Dr. A. Berlis}

Klinik für Diagnostische Radiologie und Neuroradiologie, Klinikum Augsburg

Stenglinstr. 2, 85156 Augsburg

ansgar.berlis@klinikum-augsburg.de

Danksagung. Herrn Prof. Dr med. Helmuth Forst, Chefarzt für Anästhesie und Intensivmedizin am Klinikum Augsburg, wird für seine stete Bereitschaft zur Diskussion, die bei der Abfassung des Manuskripts hilfreich war, gedankt.

\section{Einhaltung der ethischen Richtlinien}

Interessenkonflikt. A. Berlis gibt an, dass er zum Zeitpunkt der Abfassung des Manuskripts Beraterverträge mit den Firmen ev3/covidien und Microvention/ Terumo besitzt.

Dieser Beitrag beinhaltet keine Studien an Menschen oder Tieren.

\section{Literatur}

1. Abou-Chebl A, Lin R, Hussain MS et al (2010) Conscious sedation versus general anesthesia during endovascular therapy for acute anterior circulation stroke: preliminary results from a retrospective, multicenter study. Stroke 41:1175-1179

2. Anastasian ZH, Strozyk D, Meyers PM et al (2011) Radiation exposure of the anesthesiologist in the neurointerventional suite. Anesthesiology 114: 512-520
3. Berlis A (2006) Endovaskuläre Rekanalisationsverfahren bei der Behandlung des akuten Schlaganfalls. Habilitationsschrift, Albert-Ludwigs-Universität, Freiburg

4. Berlis A, Lutsep H, Barnwell S et al (2004) Mechanical thrombolysis in acute ischemic stroke with EPAR (endovascular photoacoustic recanalization). Stroke 35:1112-1116

5. Bose A, Alfke K, Henkes H et al (2008) The Penumbra system: a mechanical device for the treatment of acute stroke due to thromboembolism. AJNR Am J Neuroradiol 29:1409-1413

6. Brekenfeld C, Heinrich P, Mattle H, Schroth G (2010) General is better than local anesthesia during endovascular procedures. Stroke 41:2716-2717

7. Broderick JP, Palesch YY, Demchuk AM et al (2013) Endovascular therapy after intravenous versus tPA alone for stroke. N Engl J Med 368:893-903

8. Chimowitz MI, Lynn MJ, Derdeyn CP et al (2011) Stenting versus aggressive medical therapy for intracranial arterial stenosis. N Engl J Med 365:9931003

9. Chimowitz Ml, Lynn MJ, Howlett-Smith $\mathrm{H}$ et al (2005) Comparison of warfarin and aspirin for symptomatic intracranial arterial stenosis. N Engl J Med 352:1305-1316

10. Ciccone A, Valvassori L, Nichelatti M et al (2013) Endovascular treatment for acute ischemic stroke. N Engl J Med 368:904-913

11. Coley S, Sneade M, Clarke A et al (2012) Cerecyte coil trial: procedural safety and clinical outcome in patients with ruptured and unruptured intracranial aneurysms. AJNR Am J Neuroradiol 33:474480

12. Davis MJ, Menon BK, Baghirzada LB et al (2012) Anesthetic management and outcome in patients during endovascular therapy for acute stroke. Anesthesiology 116:396-405

13. Dorn F, Stehle S, Lockau H et al (2012) Endovascular treatment of intracerebral artery occlusions with the solitaire stent: single-centre experience with 108 recanalization procedures. Cerebrovasc Dis 34:70-77

14. Elijovich L, Randall T, Higashida MD et al (2008) Predictors and outcomes of intraprocedural rupture in patients treated for ruptured intracranial aneurysms the CARAT study. Stroke 39:1501-1506

15. Eckert B (2009) Acute stroke therapy 1981-2009. Clin Neuroradiol 1:8-19

16. Flexman AM, Donavan AL, Gelb AW (2012) Anesthetic management of patients with acute stroke. Anesthesiol Clin 30:175-190

17. Galimanis A, Jung S, Mono M-L et al (2012) Endovascular therapy of 623 patients with anterior circulation stroke. Stroke 43:1052-1057

18. Gugliemi G, Vinuela F, Dion J, Duckwiler G (1991) Electrothrombosis of saccular aneurysms via endovascular approach, part 2. Preliminary clinical results. J Neurosurg 75:8-14

19. Gugliemi G, Vinuela F, Duckwiler G et al (1992) Endovascular treatment of posterior circulation aneurysms by electrothrombosis using electrically detachable coils. J Neurosurg 77:515-524

20. Hashimoto T, Gupta DK, Young WL (2002) Interventional neuroradiology - anesthetic consderations. Anesthesiol Clin North America 20:347-359

21. Hassan AE, Chaudhry SA, Zacharatos H et al (2012) Increased risk of aspiration pneumonia and poor discharge outcome among acute ischemic stroke patients following intubation for endovascular treatment. Neurocrit Care 16:246-250 
22. John N, Mitchell P, Dowling R, Yan B (2013) Is general anaesthesia preferable to conscious sedation in the treatment of acute ischaemic stroke with intraarterial mechanical thrombectomy? A review of the literature. Neuroradiology 55:93-100

23. Jumaa MA, Zhang F, Ruiz-Ares F (2010) Comparison of safety and clinical and radiographic outcomes in endovascular acute stroke therapy fo proximal middle cerebral artery occlusion with intubation and general anesthesia versus non-intubated state. Stroke 41:1180-1184

24. Kidwell CS, Jahan R, Gornbein J et al (2013) A trial of imaging selection and endovascular treatment for ischemic stroke. N Engl J Med 368:914-923

25. Koh JS, Lee SJ, Ryu C-W, Kim SH (2012) Safety and efficacy of mechanical thrombectomy with solitaire stent retrieval for acut ischemic stroke: a systematic review. Neurointervention 7:1-9

26. Mattle HP, Arnold M, Georgiadis D et al (2008) Comparison of intra-arterial and intravenous thrombolysis for ischemic stroke with hyperdense middle cerebral artery sign (HMCAS). Stroke 39:379-383

27. McDougall CG, Spetzler RF, Zabramski JM et al (2012) The Barrow ruptured aneurysm trial. J Neurosurg 116:135-144

28. Molyneux A, Kerr R, Stratton I et al (2002) International Subarachnoid Aneurysm Trial (ISAT) of neurosurgical clipping versus endovascular coiling in 2143 patients with ruptured intracranial aneurysms: a randomised trial. Lancet 60:1267-1274

29. Molyneux AJ, Kerr RS, Birks J et al (2009) Risk of recurrent subarachnoid haemorrhage, death, or dependence and standardised mortality ratios after clipping or coiling of an intracranial aneurysm in the International Subarachnoid Aneurysm Trial (ISAT): long-term follow-up. Lancet Neurol 8:427433

30. Molyneux AJ, Kerr RS, Yu LM et al (2005) International subarachnoid trial (ISAT) of neurosurgical clipping versus endovascular coiling in 2143 patients with ruptured intracranial aneurysms: a randomised comparison of effects on survival, dependency, seizures, rebleeding, subgroups, and aneurysm occlusion. Lancet 366:809-817

31. Moret J, Cognard C, Weil A et al (1997) Reconstruction technic in the treatment of wide-neck intracranial aneurysm. Long-term angiographic and clinical results. Apropos 56 cases. J Neuroradiol 24:30-44

32. Nichols C, Carrozella J, Yeatts S (2010) Is periprocedural sedation during acute stroke therapy associated with poorer functional outcomes? J Neurointerv Surg 2:67-70

33. Nogueira RG, Lutsep HL, Gupta R et al (2012) Trevo versus Merci retrievers for thrombectomy revascularisation of large vessel occlusions in acute ischaemic sroke (TREVO 2): a randomised trial. Lancet 380:1231-1240

34. Pfefferkorn T, Holtmannspötter M, Schmidt C et al (2010) Drip, ship, and retrieve: cooperative recanalization therapy in acute basilar artery occlusion. Stroke 41:722-726

35. Preiß H, Reinartz J, Lowens S, Henkes H (2006) Anästhesiologisches Management bei neuroendovaskulären Eingriffen. Anaesthesist 55:679-692

36. Rha J-H, Saver JL (2007) The impact of recanalization on ischemic stroke outcome: a meta-analysis. Stroke 38:967-973

37. Riedel CH, Jensen U, Rohr A et al (2010) Assesment of thrombus in acute middle cerebral artery occlusion using thin-slice nonenhanced computed tomography reconstructions. Stroke 41:1659-1664
38. Risselada R, Lingsma HF, Bauer-Mehren A et al (2011) Prediction of 60 day case-fatality after aneurysmal subarachnoid haemorrhage: results from the International Subarachnoid Aneurysm Trial (ISAT). Eur J Epidemiol 25:261-266

39. Sauer H, Wankmüller H, Leschke M (2012) Blutungsmanagement, perioperatives und periprozedurales Bridging. Vorschlag praktischer Strategien für die neuen oralen Antikoagulantien. Klinikarzt 41:26-32

40. San Roman L, Obach V, Blasco J et al (2012) Single-center experience of cerebral artery thrombectomy using the TREVO device in 60 patients with acute ischemic stroke. Stroke 43:1657-1659

41. Saver JL, Jahan R, Levy El et al (2012) Solitaire flow restoration device versus the Merci Retriever in patients with acute ischaemic stroke (SWIFT): a randomised, parallel-group, non-inferority trial. Lancet 380:1241-1249

42. Serbinenko FA (1974) Balloon occlusion of saccular aneurysms of the cerebral arteries. Vopr Neirokhir 4:8-15

43. Scott RB, Eccles F, Molyneux AJ et al (2010) Improved cognitive outcomes with endovascular coiling of ruptured intracranial aneurysms. Neuropsychological outcomes from the International Subarachnoid Aneurysm Trial (ISAT). Stroke 41:1743-1747

44. Smith WS, Sung G, Saver J et al (2008) Mechanical thrombectomy for acute ischemic stroke: final results of the Multi MERCI Trial. Stroke 39:1205-1212

45. Sussmann BJ, Fitch TSP (1958) Thrombolysis with fibrinolysis in cerebral artery occlusion. J Am Med Assoc167:1705-1709

46. Taschner CA, Treier M, Schumacher M et al (2011) Mechanical thrombectomy with the Penumbra recanalization device in acute ischemic stroke. J Neuroradiol 38:47-52

47. White PM, Lewis SC, Gholkar A, Sellar et al (2011) Hydrogel-coated coils versus bare platinum coils for the endovascualr treatment of intracranial aneurysms (HELPS): a randomized controlled trial. Lancet 377:1655-1662

48. Yoon W (2011) Current update on the randomized controlled trials of intracranial aneurysms. Neurointervention 6:1-5

49. Young WL, Pile-Spellman J, Hacein-Bey L, Joshi S (1997) Invasive neuroradiologic procedures for cerebrovascular abnormalities: anesthetic considerations. Anethesiol Clin North America 15:631-653

50. Young WL (2007) Anesthesia for endovascular neurosurgery and interventional neuroradiology. Anesthesiol Clin 25:391-412

51. Zeumer H, Hacke W, Kolmann HL, Poeck K (1982) Lokale Fibrinolysetherapie bei Basilaris-Thrombose. Dtsch Med Wochenschr 107:728-731 